\title{
Mammalian Ichnopathology: a case study of Holartic Ungulates (Gomphotheriidae, Equidae, Camelidae) of the Late Pleistocene of South America. Ichnotaxomic implications
}

Cristian Oliva, Mariano Arregui

\section{Cristian Oliva}

cristianoliva78@yahoo.com.ar

Centro de Registro del Patrimonio Arqueológico y Paleontológico (CRePAP), Dirección Provincial de Museos y Preservación Patrimonial, Secretaría de Cultura de la Provincia de Buenos Aires. Calle $50 \mathrm{~N}^{\circ}$ 539. CP 1900. La Plata, Provincia de Buenos Aires, Argentina.

\section{Mariano Arregui}

YPF Tecnología S.A. Av. del Petróleo Argentino 900-1198, Berisso, CP 1923. Buenos Aires, Provincia de Buenos Aires, Argentina. Consejo Nacional de Investigaciones Científicas y Técnicas, CONICET.

BOL. SOC. GEOL. MEX. 2018

VOL. 70 NO. 2

P. $417-447$

http://dx.doi.org/10.18268/BSGM2018v70n2a10

\begin{abstract}
The analysis of paleoichnites and trackways corresponding to Holarctic ungulates, from the late Pleistocene (Lujanian Stage/Age) of Pehuen Co and Laguna del Monte tracksites (Buenos Aires Province, Argentina), allows the recognition of several clinical signs (ichnopathologies) that reflect some of the main pedal affections and alterations experienced by living members of the group. Proboscipeda australis (Aramayo and Manera de Bianco, 1987a) presents occasional fissures on its hoofprints, interpreted as fractures on the hornwal (vertical) and cracking of the sole (horizontal). Hippipeda isp. exhibits a series of morphologic anomalies in the digital impression, linked to contracted heel (transversely compressed hoof, narrowing and distal projection of talonar region, with an approximation of both heel-bulb impressions and an enlargement of the spacing between frog baseline and heel buttresses), distorted hoof (asymmetrical sole region and hoof wall, with forwardly projected internal pillar [toe-quarter junction] and anteriorly displaced external heel buttress), turkish-slipper foot (over-elongated hoofprint, showing hyper-lengthening of lateral walls [inner and outer quarters], and hypertrophy of frog and bars). Lamaichnum guanicoe Aramayo and Manera de Bianco, 1987b displays eventual evidence of horny overgrowth: inward/ subparallel (loss of bilateral symmetry, medial curving of digits, uneven sizing of toepad prints) and outward/divergent (lateral curving of digits, overexpansion of interclavular gap [with ample separation between both digital ends], enlargement and widening of the tracks, normal acquisition of a secondary heteropody). These intraspecific variations (ichnopathological characters) are often used to segregate a second llamoid ichnospecies: Lamaichnum tulipensis (Aramayo and Manera de Bianco, 1987b), herein proposed as teratologic synonym of Lamaichnum guanicoe.
\end{abstract}

Keywords: Mammal ichnopathology, Late Pleistocene, South America, Gomphotheriidae, Gamelidae, Equidae.

\section{RESUMEN}

El análisis de paleoicnitas y rastrilladas correspondientes a ungulados holárticos, provenientes del Pleistoceno tardio (piso/edad Lujanense) de los yacimientos paleoicnológicos de Pehuen Co y Laguna del Monte (Provincia de Buenos Aires, Argentina), permite el reconocimiento de diversos signos clínicos (icnopatologías) que reflejan algunas de las principales afecciones y alteraciones pedales, experimentadas por los miembros vivientes del grupo. Proboscipeda australis (Aramayo y Manera de Bianco, 1987a) presenta fisuras ocasionales en las improntas de sus pezuñas, interpretadas como fracturas del muro córneo (verticales) y agrietamientos de la suela horizontales). Hippipeda isp. exhibe una serie de anomalias morfológicas en la impresión digital, ligadas a un talón contraído (casco transversalmente comprimido, estrechamiento y proyección distal de la región talonar, con aproximación de ambas impresiones bulbares y un alargamiento del espacio entre la línea base de la ranilla y los ángulos del muro), casco distorsionado (región solar y muro asimétricos, con pilares internos proyectados hacia delante $y$ contrafuerte externo anteriormente desplazado), pie en forma de zapato turco (impresión del casco superelongada, mostrando hiper-alargamiento de los cuartos interno y externo e hipertrofia de ranilla y barras). Lamaichnum guanicoe Aramayo y Manera de Bianco, $1987 b$ muestra eventuales evidencias de sobrecrecimiento córneo: subparalelo/interno (pérdida de simetría bilateral, curvamiento medial de los dígitos, desigual desarrollo de las almohadillas digitales) y divergente/externo (curvamiento lateral de los dígitos, expansión del espacio interdigital [con amplia separación entre ambos extremos digitales], alargamiento y ensanchamiento de las huellas, normal adquisición de una heteropodia secundaria). Estas variaciones intraespecificas (caracteres icnopatológicos) son a menudo utilizadas para segregar una segunda icnoespecie: Lamaichnum tulipensis (Aramayo y Manera de Bianco, 1987b), aquí propuesta como sinónimo teratológico de Lamaichnum guanicoe.

Palabras clave: Icnopatología mamíferos, Pleistoceno tardio, Sudamérica, Gomphotheriidae, Camelidae, Equidae. 


\section{Introduction}

Paleopathology, the study of ancient diseases (Rothschild and Shelton, 1992; Rothschild and Tanke, 1992; Rothschild and Martin, 1993; Waldron, 2009), has experienced notable progress in the last three decades, mainly from paleoanthropological (Ortner and Putschar, 1980; Aufderheide and Rodriguez-Martin, 1998; Isidro and Malgosa, 2003; Ortner, 2003a, 2003b), zooarqueological (Baker and Brothwell, 1980; Bartosiewicz, 2008) and paleontological viewpoints (Rothschild, 1988, 1990, 1997; Tanke, 1989; Ferigolo, 1992, 2007; Rothschild et al., 1997, 2001, 2012; Henriques et al., 1998; Marshall et al., 1998; Rothschild and Molnar, 1988; Sullivan et al., 2000; Tanke and Currie, 2000; Molnar, 2001; Hanna, 2002; Tanke and Rothschild, 2002, 2010, 2015; Bennett, 2003; Rothschild and Tanke, 2005; Aramayo, 2006; Rothschild and Martin, 2006; Farke and O'Connor, 2007; Lü et al., 2007; Druetta, 2009, 2012; Xing et al., 2009, 2015; Bell, 2010; Rega et al., 2010; Bell et al., 2011; Barbosa et al., 2013, 2014a, 2014b, 2017a, 2017b; Ferreira et al., 2013; Barbosa and Luna, 2014).

In contrast, the advances in ichnopathology (paleopathology based on the fossil-tracks analysis) are much more restricted and specifically focused on dinosaurs (see Lockley et al., 1994; Tanke and Rothschild, 2002; McCrea et al., 2014, 2015).

In this regard, although this specialty has developed parallel to conventional ichnology since the mid-nineteenth century, its evolution was comparatively lesser, headed by sporadic contributions wherein a scarce number of anomalous records, from the Mesozoic of Northern Hemisphere, were described: Late Triassic-Early Jurassic (Hitchcock, 1844, 1858; Abel, 1935; Lull, 1953) and Late Cretaceous (McCrea et al., 2014, 2015; Tanke and Rothschild, 2015) of North America; Triassic s. 1. (Tucker and Burchette, 1977) and Late Jurassic (Dantas et al., 1994; Avanzini et al., 2008) of Europe; Middle Jurassic of Africa JJenny and Josen, 1982; Ishigaki, 1986, 1988) and Late Cretaceous of Asia (Currie et al., 2003; McCrea et al., 2015).

This is probably due to the high difficulty that imply the differentiation of pathological signs in footprints and trails, which reflect the influence of several extrinsic agents (e.g., nature of the track-bearing substrate, behaviour of the trackmaker, taphonomical factors) (see McCrea et al., 2015); made even worse in the cases where there are no modern species for comparison.

In this paper we present the results obtained from the paleopathological study of fossil tracks and trackways related to Holarctic ungulates (equids, camelids and gomphotherids), from the Late Pleistocene of Argentina (South America), and discuss the ichnopathological and ichnotaxonomical implications of these findings.

\section{Materials and methods}

The ichnopathological analysis was made on specimens assignable to the ichnospecies Proboscipeda australis (Aramayo and Manera de Bianco, 1987a); Lamaichnum guanicoe (Aramayo and Manera de Bianco, 1987b); Lamaichnum tulipensis (Aramayo and Manera de Bianco, 1987b); and Hippipeda isp.; from the Late Pleistocene (Lujanian Stage/Age) of Pehuen Co (Partido de Coronel Rosales) and Laguna del Monte (Partido de Guaminí) tracksites, Buenos Aires Province, Argentina (Figure 1).

The differentiation of pathologic footprints was based on the detection of potential clinical signs (related to any of the limb affections known for modern ungulates); having previously discarded other types of intraspecific variations (intramorphological changes) and/or secondary alterations linked to external factors (extramorphological characters) (e.g., nature of the substrate, mechanical disturbance, presence of foreign objects, infauna influence; see McCrea et al., 2015).

The recognition of the ungual diseases has been made indirectly, through interpretation of 


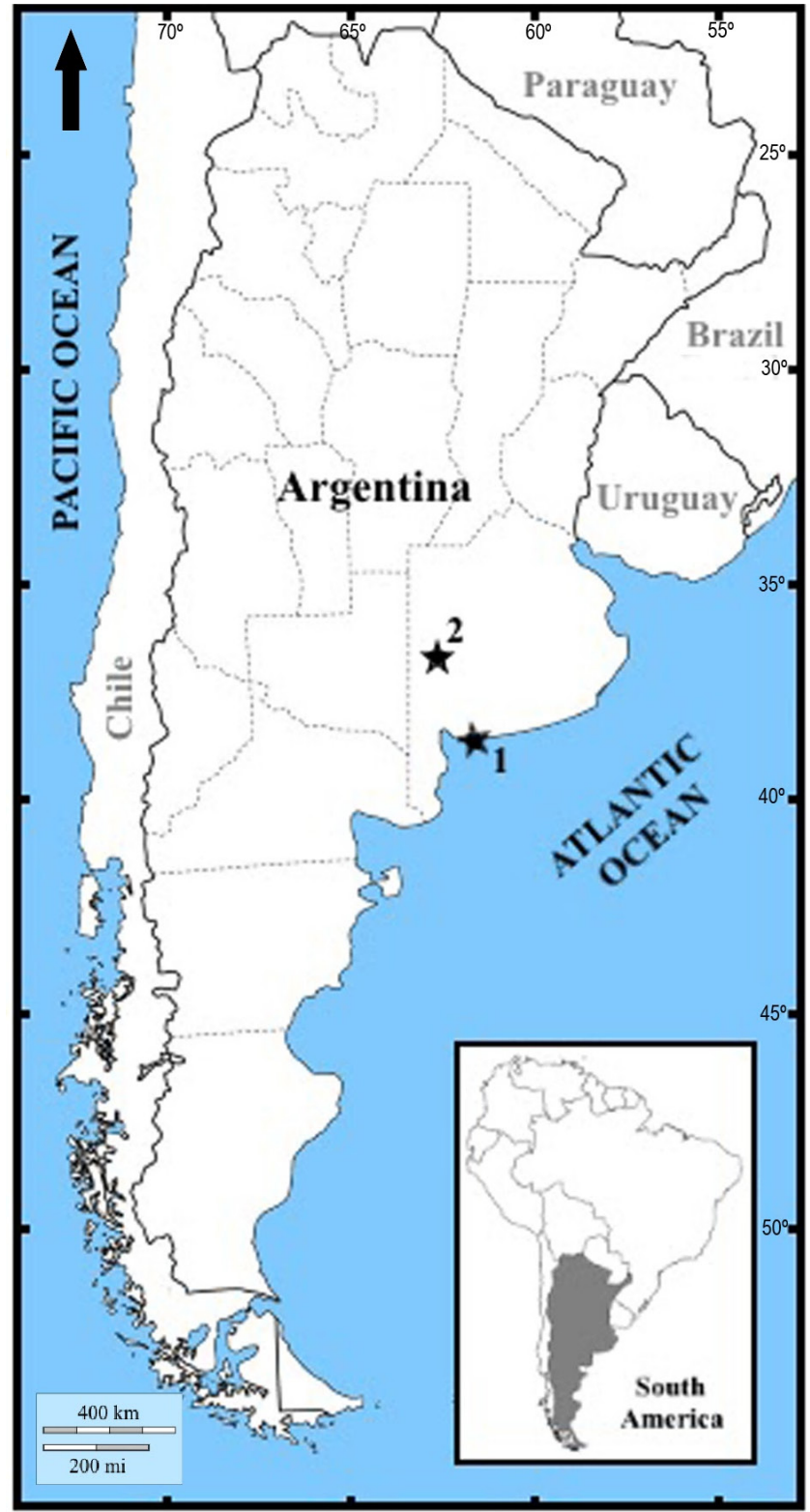

Figure 1 Geographic location of provenance tracksites of the study material. (1) Pehuen Co (Coronel Rosales); (2) Laguna del Monte (Guaminí); Buenos Aires Province, Argentina.

veterinary literature, and/or directly, by consultation of specialists (rural and zoo veterinarian, specialized breeders) and comparison with autopods of living members of the group: Loxodonta africana Blumenbach, 1797 (African elephant); Elephas maximus Linnaeus, 1758 (Asian elephant); Equus ferus caballus Linnaeus, 1758 (domestic horse); Equus africanus asinus Linnaeus, 1758 (domestic donkey); Equus quagga Boddaert, 1785 (plains zebra); Lama guanicoe Müller, 1776 (guanaco); Lama glama Linnaeus, 1758 (llama); Vicugna vicugna Molina, 1782 (vicugna); Vicugna pacos Linnaeus, 1758 (alpaca).

Following the modern veterinary tendencies, the podal disorders were classified and grouped according into clavular (ungular); solar; bulbar/ palmar/talonar; interdigital; and phalangeal (osteological) subtypes.

Most of the study material was examined in the field (LDM: Laguna del Monte Tracksite; PCT: Pehuen Co Tracksite), while the remaining specimens reside in the Paleoichnological Collections of the Museums "Marcelino Freyre" (Guaminí) (MG-Pic) and "Carlos Darwin" (Punta Alta) (MD-YPI).

Institutional abbreviations. LDM: Laguna del Monte Tracksite (Guaminí, Partido de Guaminí). MD-YPI: Museo Darwin; Colección Yacimiento Paleoicnológico (Punta Alta, Partido de Coronel Rosales). MG-Pic: Museo Municipal Marcelino Freyre; Colección Paleoicnológica (Guaminí, Partido de Guaminí). PCT: Pehuen Co Tracksite (Pehuen Co, Partido de Coronel Rosales); Buenos Aires Province, Argentina.

\section{Podal diseases on living ungulates}

Because of their unguligrade/subunguligrade nature, ungulates are particularly susceptible to a broad range of podal diseases, from simple lesions and trauma (e.g., penetrating injuries, cutting wounds, contusions, foreign bodies) to a wide variety of phalangeal/osteological affections (osteopathies, bone lesions) and complications (e.g., cracking, overgrowth, infections) in the clavular (ungular/solar) and palmar/talonar (heel bulbs, digital pads, foot pad) regions.

In this regard, a great number of foot disorders have been recognized and systematically studied for several hoofed mammals: Proboscidea Owen, 1848 (Elephantidae Gray, 1821); Perissodactyla Owen, 1848 (Equidae Gray, 1821; Rhinocerotidae Gray, 1821; Tapiridae Burnett, 
1829); Artiodactyla Owen, 1848 (Camelidae Gray, 1821; Cervidae Gray, 1821; Bobidae Gray, 1821; Giraffidae Gray, 1821; Hippopotamidae Gray, 1821; Suidae Gray, 1821; Tayassuidae Palmer, 1897), either on wild or captive animals (Fowler, 1978, 1980).

Among the main clinical conditions detected on these mammalian lineages can be mentioned: (1) talonar/palmar/bulbar: heel erosion, heel wart (talonar hyperplasia), heel abscess, heel necrosis, greasy heel, crushed heel, sheared heel; (2) solar: solar injuries and trauma (e.g., punctured sole, penetrating wounds, solar hemorrhage), dropped sole, solar callus, solar pressure, aseptic pododermatitis (laminitis or pododermatitis aseptica diffusa; solar ulceration or pododermatitis circumpscripta), infectious or bacterial pododermatitis (purulent, suppurative, necrotic, gangrenous); (3) clavular: proximal dermic (e.g., toe ulceration, digital and verrucous dermatitis, coronary phlegmon, flexor-zone abscess, exungulation, split toenails), hoofwall alterations (e.g., hoofwall cracks, fissures, and trauma; overgrown horn), white-line diseases (inflammation, hypertrophy and abscessation of white line, gravel, seedy toe);

(4) interdigital: postdigital and interdigital dermatitis, interdigital phlegmon, necrobacillosis, and hyperplasia; and (5) osteological: osteomyelitis, arthritis, heterotopic ossification, subluxations and luxations, osseous fractures and fissures.

The cited pathologies are common to all ungulates. However, their etiology, clinical pattern, evolution, and degrees of incidence and gravity vary notably from one group to another.

The neospecies of proboscidean, Loxodonta africana (African elephant) and Elephas maximus (Asian elephant), as other foot-padded ungulates (tapirs, rhinoceros, hippopotamus), are particularly susceptible to the overgrowth, cracking, and infection (pododermatitis traumatica or abscess; pododermatitis chronica suppurativa or foot rot) of the heel cushion (Kuntze, 1972, 1980; Fowler, 1980, 2001; von Houwald, 2001; Benz, 2005) (Figure 2A). They are also affected by a series of moderate (overgrown horn, horn-wall cracking) to severe (laminitis, exungulation) hoof illnesses (see Kuntze, 1980; Fowler, 1993; Mikota et al., 1994; West, 2001; Benz, 2005) (Figure 2B-D).

Horny overdevelopment constitutes the most frequent limb disease (along with the ulcerative pododermatitis of digital pads) in modern camelids, such as the species of the South American genera Lama Cuvier, 1800 (Lama guanicoe [wild]; Lama glama [domestic]) and Vicugna Miller, 1924 (Vicugna vicugna [wild]); Vicugna pacos [domestic]).

This condition is distinguished by the hypertrophy and consequent alteration (elongation, curving, and deviation) of the toenails, which depart from their natural position (forwardly directed, aligned with the digital axis) (Figure 3A) to be inwardly (subparallel) (Figure 3B), medially (convergent) (Figure 3C), or outwardly (divergent) (Figure 3D) oriented.

The unguals in digitigrade camelids are nonweight bearing, with the entire body weight falling on the toe cushions, unlike semi-unguligrade and unguligrade mammals, in which the hooves support most of the load. However, healthy nails remain crucial during the traction and propulsion of the individuals. For this reason, while horny overgrowth does not constitute necessarily a chronic problem in llamoids, except if generates laminitis, it does affect at long-term the locomotion and lateral stability of these animals.

Linked with an inadequate corneous wear and a disturbance in the cuticular keratogenesis, horny overdevelopment aggravates in unguligrade equids: Equus ferus caballus (domestic horse); Equus africanus asinus (domestic donkey); Equus quagga (plains zebra). Initially manifested as an over-elevation, thickening, and cracking of the hoofwall (Figure 4A), this disorder can evolve towards a strong deformation of the hooves, which acquire a "turkish slipper" (Figure 4B) and/or "helical" (Figure 4C) appearance.

The high and unstable hooves generated in the first case may potentially collapse, causing the separation of outer wall and hoof capsule, and 

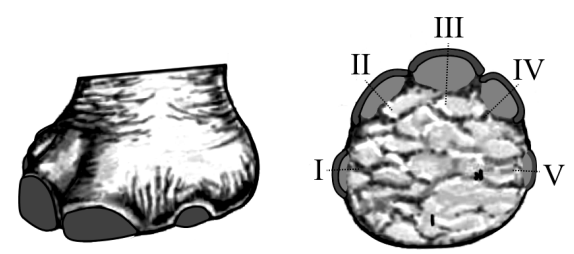

A
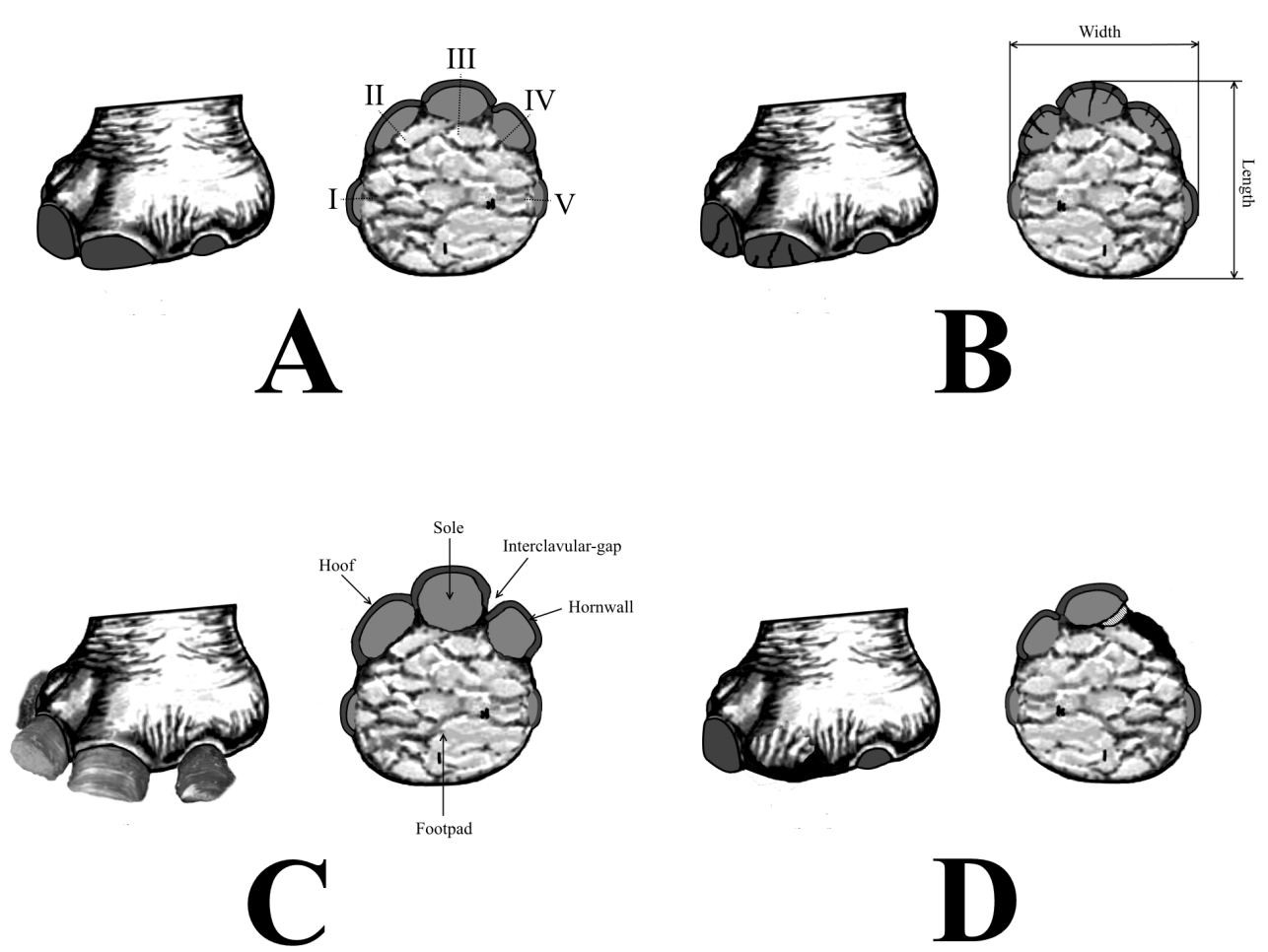

Figure 2 Principal podal diseases in living elephants (Proboscidea, Elephantidae, Elephantinae); lateral and palmar/plantar views (schematic). (A) Pododermatitis; (B) hornwall cracking; (C) overgrown horn; (D) exungulation. Based on Benz (2005).

thereby facilitating the weakening and fissuration of the hoofwall, besides the occurrence of bacterial/fungal infections and abscesses. This condition, known as seedy toe, white line disease, or wall separation, is characterized by a horadation of the hoofwall and widening of the white line, with the development of minor slits to extensive cavities between both structures and the formation of a grey crumbly residue as a result of their infectious necrosis.

The second state, typically associated with laminitis, comprises the anterior projection and dorsal bending of the hoofwall (corneous hypergrowth), a frontal stretching of the white line and an aberrant overdevelopment of the bars.

The laminitis constitutes an inflammatory hoof-membranes disease that causes the progressive degeneration, separation, and necrosis of the laminar corion, with a consequent detachment, rotation, and sinking of the distal phalanx into the horny cover of the foot, by action of the force exerted by the corporal weight of the horse.
An acute clinical pattern of this pathology embraces a series of inflammatory processes and deep circulatory changes into the hooves, which, although generating intense pain and feverishness of toe and coronet, do not result into podal deformation.

Conversely, a chronic clinical pattern, involving the loss of the laminar support that positions the distal phalanx within the hoof, causes significant alterations of the autopodium: (1) drop, flattening, thinning, and weakening of the sole (dropped sole), with perforation of the same (puncture sole) in severe cases of rotation and descent of the ungual bone; (2) hypertrophy and coronal tearing of the white line; (3) distal overdevelopment of the hoof, with antero-dorsal projection of the toe (turkish slipper foot) and/or spiraling overgrowth of the hoofwall (helical foot); (4) abnormal development of coronary bands, with emergence of thick transversal grooves along the wall.

In both clinical phases, the physical signs are accompanied by important behavioral changes: 

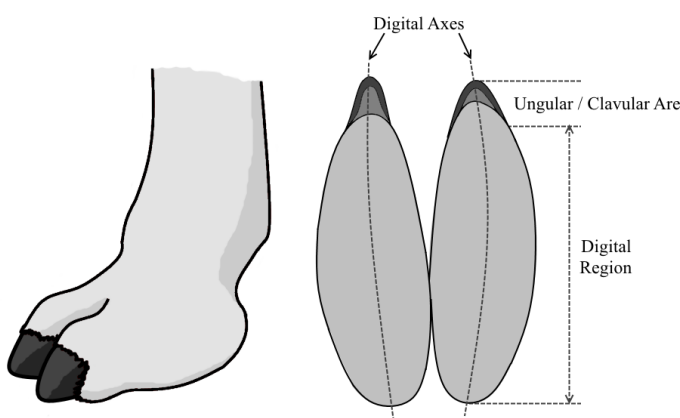

A
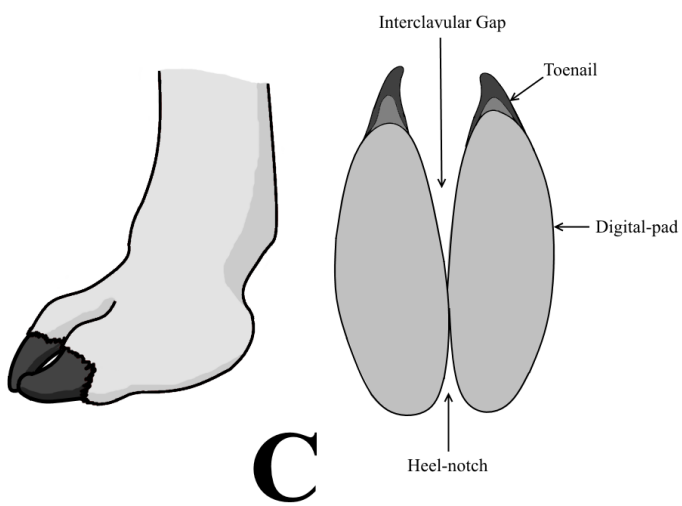

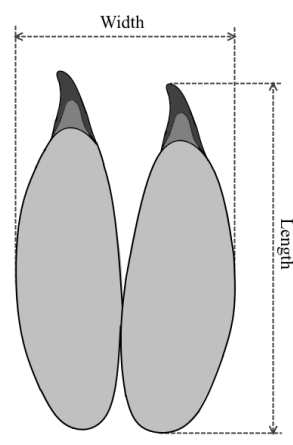

B
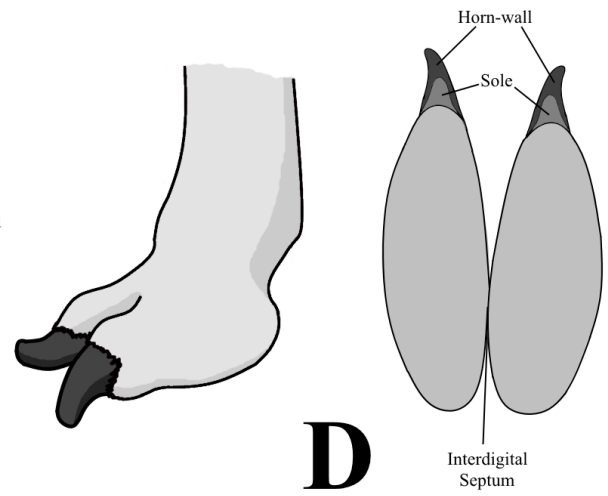

Figure 3 Principal clavular alterations in living Ilamoids (Artiodactyla, Camelidae, Lamini); left/lateral and palmar/plantar views (schematic). Footprints showing: (A) natural growth; (B) inward/subparallel; (C) medial/convergent; (D) outward/divergent, toenail overgrowths.

(1) recurrent recline and resistance to walking; (2) abnormal gait (e.g., measure, short and fast steps, frequent limping march); and (3) antalgic posture (forwardly directed limbs).

The normal or balanced equine hooves (unaltered autopodes) are almost bisymmetric and present transversely aligned heel buttresses, disposed approximately at the same level as the frog baseline and heel bulbs (Figure 5A).

In these unaltered feet, only the weight-bearing hoofwall exerts pressure on the ground surface, with a passive contact of sole and frog; this latter structure, acting as circulatory pump and shock dissipater for the hoof.

Although autopodes with contracted heel maintain a bilateral symmetry, they experience a series of modifications in the hoof (dorsoventral expansion and steepness of the hornwall) and the heel (narrowing, elevation and distal projection of the talonar area, with an approximation of both heel bulbs, atrophy of the frog and enlargement of distance between frog baseline and heel buttresses). These changes are usually accompanied by a lateral compression and deformation of the sole, with an increase of the spacing between frog apex and hornwall (Figure 5B).

On the other hand, feet experiencing bilateral distortion of the ungular region (distorted hoof) display a strong pedal asymmetry, with uneven development of medial (long and flared) and lateral (short and upright) walls into the hoof, forwardly projected internal pillars (toe-quarter junctions), and anteriorly displaced external heel-buttresses (Figure 5C). This type of hoof anomaly implies a loss of medial/lateral balance in the foot, as well as an imbalance in the support 


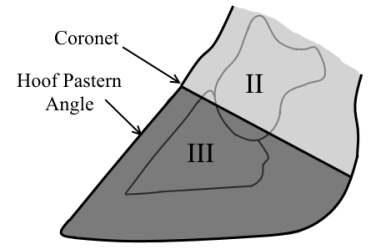

A

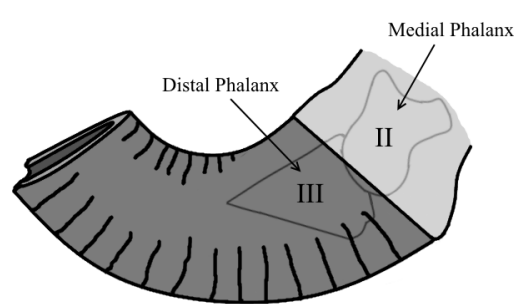

B

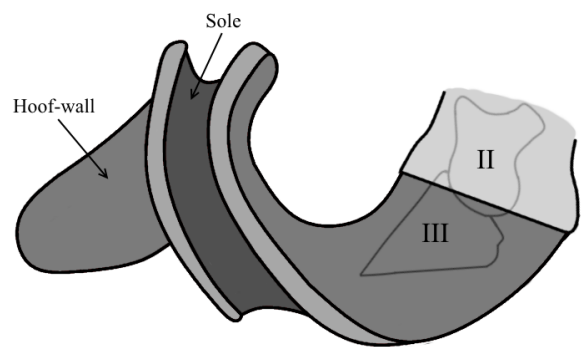

C

Figure 4 Types of horny overdevelopment in living equines (Perissodactyla; Equidae; Equini); lateral views (schematic). (A) overelevated hoof (incipient overgrowth); (B) turkish-slipper hoof (acute hypertrophic overgrowth); (C) helical hoof (chronic hypertrophic overgrowth).

of the body weight (exerting on the inner quarter a greater strain than the outer quarter).

Besides the described alterations and afflictions, numerous hoof diseases are identified in living equids. These include (1) bulbar: crushed heels, sheared heels, greasy heels (mud rash, mud fever), heel abscess (infected corn), foot gangrene; (2) ungular: quarter cracks, coronary band lesions (bruising, injuries, lacerations, traumas), coronet concussion; and (3) sole and frog: solar pressure, lesions (injuries, puncture/penetrating wounds, traumas), underrun sole/frog, haemoglobin staining at the white line zone, sarcoid, phycomycosis (pythiosis), canker, thrush, keratoma (see Pollitt, 1995).

\section{Systematic ichnology}

Ichnodivision Vertebratichnia Vialov, 1966

Ichnoclass Mammalipedia Vialov, 1966

Ichnorder Proboscipedida Remeika et al., 1995

Morphofamily Gomphotheriipedidae Remeika, 2001

Ichnogenus Proboscipeda Panin and Avram, 1962

Type Ichnospecies. Proboscipeda enigmatica Panin and Avram, 1962.

Proboscipeda australis (Aramayo and Manera de Bianco, 1987a)

(Table 1; Figure 6)
Stegomastodonichnum australis Aramayo and Manera de Bianco, 1987a (Aramayo and Manera de Bianco, 1987a, 2000; Lucas et al., 2007; Manera de Bianco et al., 2010; Oliva et al., 2013; Aramayo et al., 2015).

Study material. Pehuen Co; PCT-I: trackway of seven footprints (holotype) (Figure 6A-B); MPA87-56-I-E: plaster cast of footprint belonging to the holotype (Figure 6C) (Aramayo and Manera de Bianco, 1987a; Aramayo et al., 2015); PGT-II: forefoot imprint (Figure 6G) corresponding to a trackway of three paleoichnites (Aramayo et al., 2015). Laguna del Monte; LDM- $\mathrm{S}_{2} \mathrm{I}_{22}$ : isolated forefoot imprint (Figure 6D); LDM-S $\mathrm{S}_{7}$ : couple of overstepped forefoot and hindfoot imprints (Figure 6E); LDM-S $\mathrm{T}_{6}$ : partial trackway of four incomplete paleoichnites (Figure 6F); LDM-S $\mathrm{T}_{7}$ : trackway of four overlapped forefoot and rearfoot imprints (Figure 6H) (Oliva et al., 2013; Oliva and Arregui, 2016); all specimens remain in situ (excepting MPA-87-56-I-E).

Extended diagnosis. (modified from Aramayo et al., 2015). Large subcircular to subovoidal footprints (length: 230 - $270 \mathrm{~mm}$; width: $230-300$ $\mathrm{mm}$ ), of semi-plantigrade type, related to medium-sized proboscidean of the Gomphotheriidae Family. Moderate heteropody, with manus (wider than long, with length/width ratio < 1) slightly larger than pes (isodiametric or longer than wide, with length/width ratio $\geq 1$ ). Three to five digital impressions, tangentially arranged along 

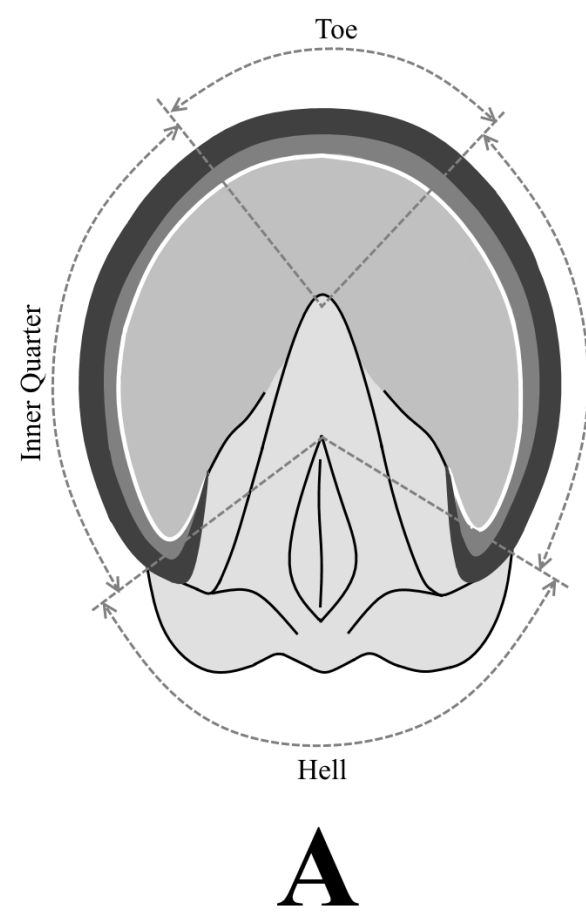
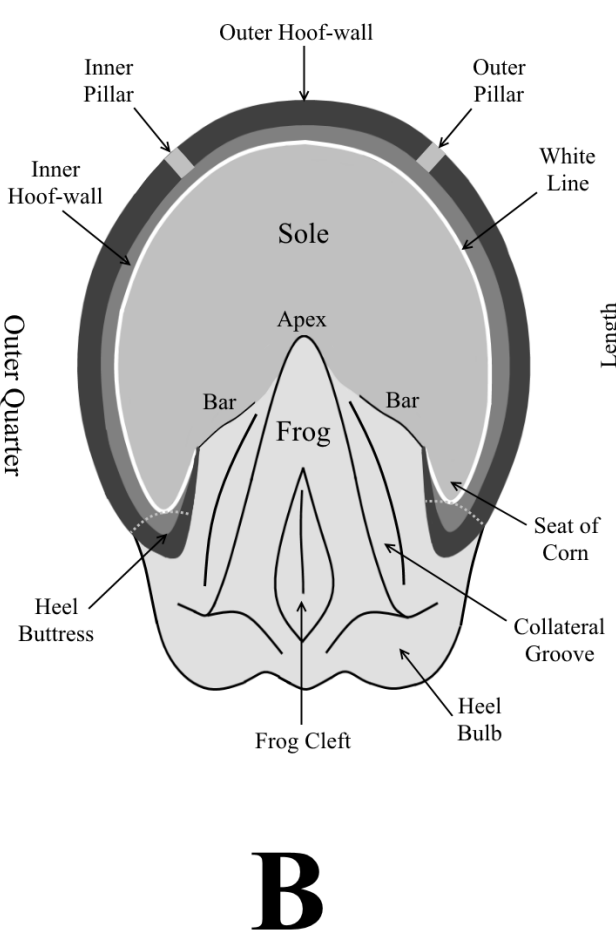
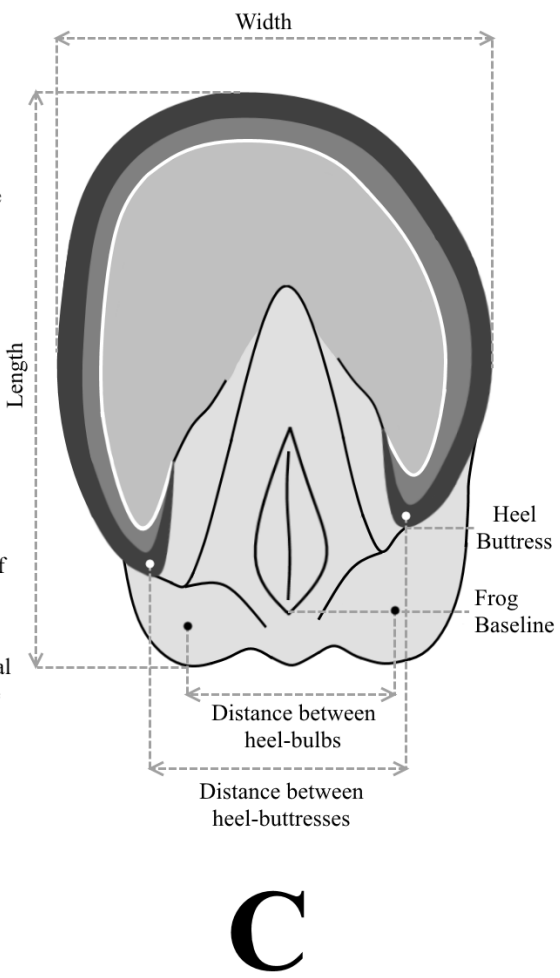

Figure 5 Principal hoof alterations in modern equines (Perissodactyla; Equidae; Equini); palmar/plantar views (schematic). (A) Balanced hoof (healthy foot); (B) unbalanced hoof, with compressed heel; (C) distorted hoof, with bilateral asymmetry.

the anterior margin of the track, appearing as a series of slight undulations and/or more or less differentiated hoofprints. Broad and rounded metapodial cushion (footpad print), conforming a wide flattened surface that encompasses the most part of the ichnite, either smooth or irregularly ornamented (pockmarked textured, cracked, and wrinkled), according the trace being poorly to well preserved, respectively.

Geographic and chronostratigraphic distribution. Late Pleistocene of Buenos Aires Province, Argentina (Aramayo and Manera de Bianco, 1987a, 2000; Oliva et al., 2013; Aramayo et al., 2015).

\section{Possible trackmaker. Stegomastodon} platensis (Ameghino, 1888) (= Notiomastodon platensis) (Proboscidea Owen, 1848; Gomphotheriidae Cabrera, 1929; Anancinae Hay, 1922).

Description. Proboscipeda australis exhibits the archetypal morphology of elephantine track that defines the ichnogenus Proboscipeda; characterized by its great size, hemispherical constitution and structural simplicity (see Lucas et al., 2007; McNeil et al., 2007; Aramayo et al., 2015).

Unaltered footprints. Constitute large and deeply imprinted paleoichnites of roughly circular/ovoidal outline, which are bilaterally symmetrical, almost isodiametrical and usually surrounded by a noticeable annular ridge (marginal rim) (Figure 6A-F).

Anatomical differences between forefoot and hindfoot impressions are nonexistent. Nevertheless the manus (length: $240-290 \mathrm{~mm}$; width: $250-300$ $\mathrm{mm}$ ), typically wider than long (length/width ratio: $0.80-0.96)$, is invariably larger than the pes (length: $230-270 \mathrm{~mm}$; width: $230-250 \mathrm{~mm}$ ), isodiametric and/or longer than wide (length/ width ratio: 1.00 - 1.10) (Table 1).

These footprints possess a broad flattened palmar/ talonar region, interpreted as the imprint generated by a thick metapodial-cushion (footpad-print), whose surface can be pockmarked (cracked and 

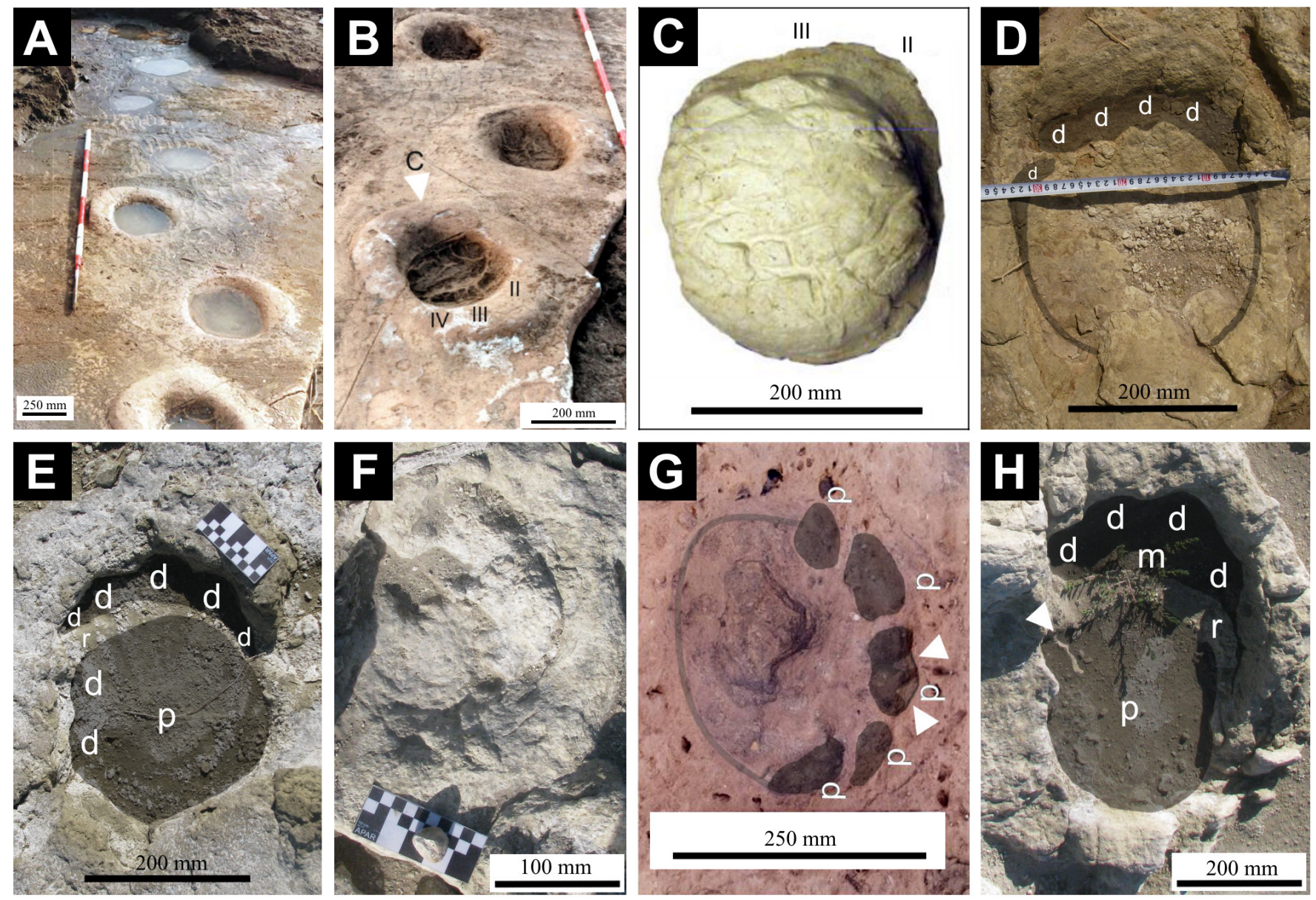

Figure 6 Gomphotherid paleoichnites [Proboscipeda australis (Aramayo and Manera de Bianco, 1987a)]. Unaltered footprints (healthy feet): (A-C) holotype specimen; in (B) the white arrow indicates the paleoichnite that served as a cast for (C); (D) isolated forefoot imprint; (E) couple of overstepped forefoot and hindfoot tracks; $(F)$ better preserved footprint of a partial trackway. Altered footprint (unhealthy feet): (G) forefoot imprint (belonging to a partial trackway) and (H) couple of overlapped forefoot and rearfoot tracks; both displaying evidence of hornwall fractures and sole fissures (white arrows ). Abbreviations: d: undifferentiated digital impressions; m: manus (forefoot); p: pes (hindfoot); r: rim (marginal sedimentary ridge); II to IV: hoofprints. A-C and G, modified from Aramayo et al. (2015).

wrinkled) (Figure $6 \mathrm{~A}-\mathrm{G}$ ) or featureless (smooth) (Figure 6D-F) depending on the preservational state.

On better-preserved specimens, it is possible to appreciate a series of small digital impressions, disposed in close hemicircular pattern along the anterior margin of the track (with their longer transverse axis arranging tangentially to the footpad print).

Similar in size and shape, these toe imprints can appear as slight undulations (Figure 6D-F, H) and/or more or less differentiated, subcircular to subrectangular shaped, hoofprints (Figure 6B-C, G).

In both cases, the nearly aligned central toes (anteriorly pointed; subparallely disposed) are forwardmost projected than the slightly delayed lateral digits (antero-laterally directed; somewhat divergent).

Contrary to what Aramayo et al. (2015) observed, the number of hooves in Proboscipeda australis may be indistinctly three to five, either in manus and pes, such as in modern species of proboscidean (see Ramsay and Henry, 2001; Benz, 2005). 
Table 1. Footprint measurements of South American, Late Pleistocene, proboscidean-tracks: Proboscipeda australis (Aramayo and Manera de Bianco, 1987a). L: track length; W: track width; L/W: length/width ratio.

\begin{tabular}{|c|c|c|c|c|c|}
\hline \multirow{2}{*}{\multicolumn{2}{|c|}{$\begin{array}{c}\text { Designation } \\
\text { (acronym) }\end{array}$}} & \multirow{2}{*}{$\begin{array}{l}\text { Referred } \\
\text { Imprint }\end{array}$} & \multicolumn{3}{|c|}{ Measurements (mm) } \\
\hline & & & $\mathrm{L}$ & W & $\mathrm{L} / \mathrm{W}$ \\
\hline \multicolumn{2}{|c|}{$\mathrm{LDM}_{-} \mathrm{S}_{1} \mathrm{~T}_{6}$} & Hindfoot & 235 & 230 & 1.02 \\
\hline \multirow{2}{*}{ LDM-S $\mathrm{T}_{7}$} & $\mathrm{I}_{1}$ & Forefoot & - & 250 & - \\
\hline & $\mathrm{I}_{2}$ & Hindfoot & $>240$ & 230 & - \\
\hline \multirow{2}{*}{ LDM-S $S_{7}$} & $\mathrm{I}_{1}$ & Forefoot & - & 250 & - \\
\hline & $\mathrm{I}_{2}$ & Hindfoot & 230 & 250 & 1.08 \\
\hline \multicolumn{2}{|c|}{$\mathrm{LDM}-\mathrm{S}_{2} \mathrm{I}_{22}$} & Forefoot & 290 & 300 & 0.97 \\
\hline \multicolumn{2}{|c|}{ PCT-I (MPA-87-56-I-E)* } & Hindfoot & 270 & 250 & 1.08 \\
\hline & $\mathrm{I}_{1}$ & Forefoot & 240 & 280 & 0.85 \\
\hline \multirow[t]{2}{*}{ PCT-II* } & $\mathrm{I}_{2}$ & Forefoot & 240 & 300 & 0.8 \\
\hline & $\mathrm{I}_{3}$ & Hindfoot & 240 & 240 & 1 \\
\hline \multirow{2}{*}{\multicolumn{2}{|c|}{ Ichnospecifical Range** }} & Forefoot & $240-290$ & $230-300$ & $0.8-0.97$ \\
\hline & & Hindfoot & $230-270$ & $210-240$ & $1.0-1.10$ \\
\hline
\end{tabular}

*Compiled from Aramayo and Manera de Bianco, 1987a, 2010; Aramayo et al., 2015.

*Extended from: Aramayo and Manera de Bianco, 1987a; Aramayo et al., 2015.

Pathological footprints. The irregular skin texture, characteristic of the footpad print of proboscidean tracks (both extinct as extant), precludes notably the correct recognition and diagnosis of palmar/plantar diseases.

Additionally, these footprints rarely preserve the digital impressions (hoofprints), greatly hindering the identification of ungual disorders.

However, some well preserved isolated specimens of Proboscipeda australis exhibit inbound peripheral ridges on its hoofprints (interpreted as vertical fissures on the hornwall) that prolong inward, towards the sole region (solar cracking) (Figure $6 \mathrm{G}-\mathrm{H})$.

Leaving aside these scarce examples, the available paleoichnological record does not provide evidence of the occurrence of other clavular alterations in fossil elephants, unlike the numerous pathologies documented for living members of the group (e.g., overgrown horn, laminitis, exungulation).

Order Artiodactypedida Vialov, 1966 Morphofamily Pecoripedidae Remeika et al., 1995
Ichnogenus Lamaichnum Aramayo and Manera de Bianco, 1987b

Type ichnospecies. Lamaichnum guanicoe Aramayo and Manera de Bianco, 1987b.

Lamaichnum guanicoe Aramayo and Manera de Bianco, 1987b

(tables 2-3; figures 7-8)

Lamaichnum alfi Sarjeant and Reynolds, 1999; Lamaichnum obliquiclavum Sarjeant and Reynolds, 1999; Lamaichnum etoromorphum Sarjeant and Reynolds, 1999; Lamaichnum borregoensis Remeika, 1999; Lamaichnum tulipensis (Aramayo and Manera de Bianco, 1987b) Aramayo et al., 2015 (= Megalamaichnum tulipensis Aramayo and Manera de Bianco, 1987b) nov. sin. (teratological synonym).

Study material. (referred to Lamaichnum guanicoe). Pehuen Co; PCT-III: trackway of eleven footprints (holotype) (Aramayo and Manera de Bianco, 1987b); PGT-IV: twenty-one footprints, conforming several trackways (neotype) (figures 

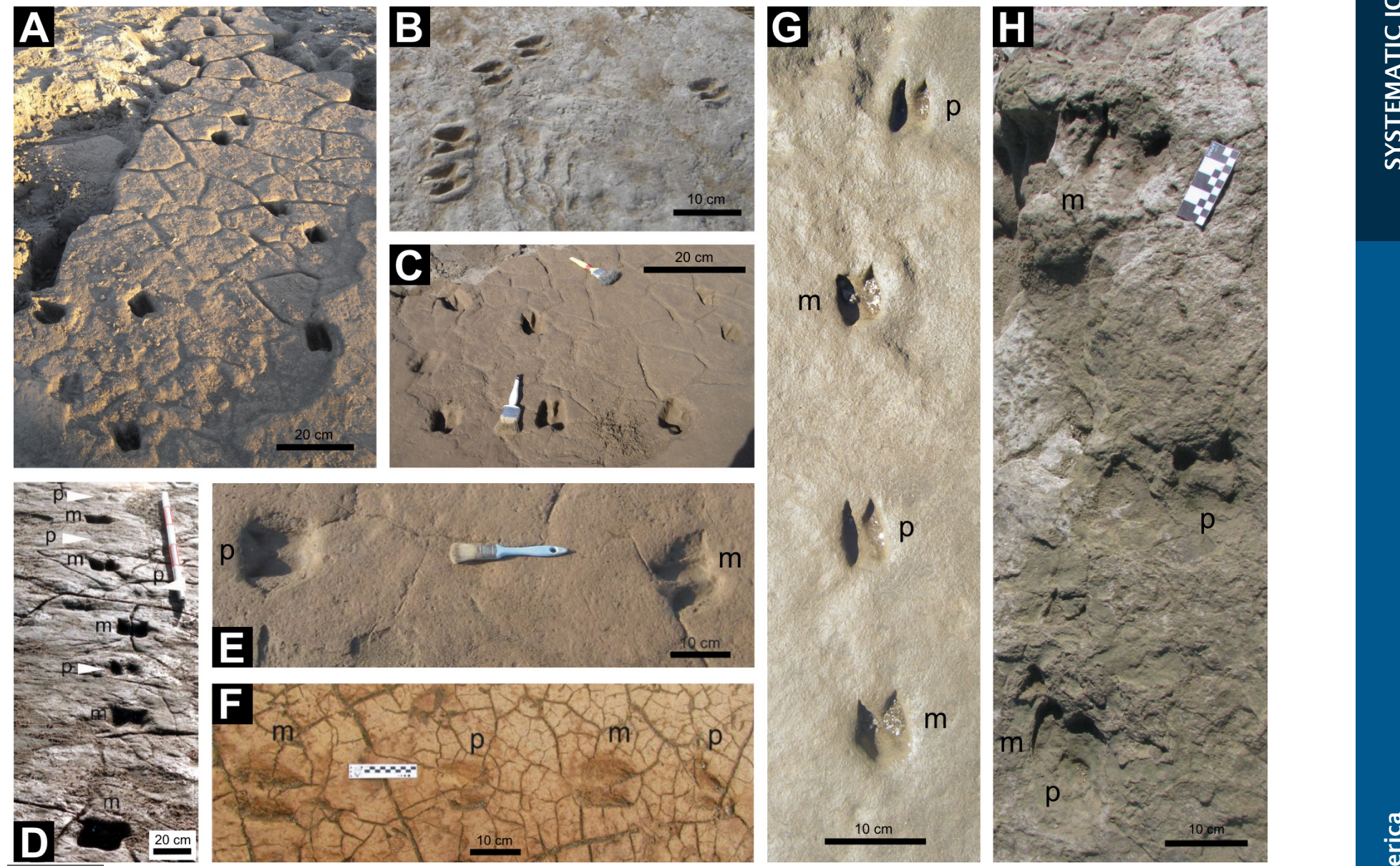

Figure 7 Llamoid fossil trackways (Lamaichnum guanicoe Aramayo and Manera de Bianco, 1987a) composed by A-C: unaltered footprints (healthy feet) and D-H: altered footprints (unhealthy feet), showing signs of toenail overgrowths. Abbreviations: m: manus (forefoot); p: pes (hindfoot). A-B, D, and F, modified from Aramayo et al. (2015); C and E, modified from Manera de Bianco and Aramayo (2013).

7A, 7C, 8E); MD-YPI-05-15: silicone rubber cast of the anterior specimen; PGT-V: around fifty footprints (Figure 7B) [sharing a trample surface with other tracks of bird (Gruipeda isp.) and mammals (Canipeda isp.; Eumacrauchenichnus patachonicus Aramayo and Manera de Bianco, 1987a)]; MD-YPI-15-01: silicone rubber cast of this bioturbed area (Aramayo et al., 2015); PGT-VI: trackway of seven footprints (figures $7 \mathrm{G}, 8 \mathrm{~A}$ ) (unpublished). Laguna del Monte; LDM- $\mathrm{S}_{1} \mathrm{I}_{24}$; LDM-S $\mathrm{I}_{25} ;$ LDM-S $\mathrm{I}_{40} ;$ LDM-S $\mathrm{I}_{41} ;$ LDM-S $\mathrm{I}_{42} ;$ LDM-S $\mathrm{I}_{43} ;$ LDM-S $\mathrm{I}_{44} ;$ LDM-S $\mathrm{I}_{45} ;$ LDM-S $\mathrm{I}_{52}$; LDM-S $\mathrm{I}_{73} ;$ LDM-S $\mathrm{I}_{78} ;$ LDM-S $\mathrm{I}_{79} ;$ LDM-S $\mathrm{I}_{82} ;$

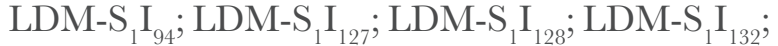
LDM-S $_{1} \mathrm{I}_{134} ;$ LDM-S $_{1} \mathrm{I}_{176}$ (Figure 8B); LDM-S $\mathrm{I}_{177}$ (Figure 8C); LDM-S $\mathrm{I}_{178}$ (Figure 8F); LDM-S $\mathrm{I}_{179}$
(Figure 8G); LDM-S $\mathrm{I}_{180}$ (Figure 8I); LDM-S $\mathrm{I}_{181}$ (Figure 8K); LDM-S $\mathrm{I}_{5}$; LDM-S $\mathrm{I}_{6}$ (Figure 8J); LDM-S $\mathrm{I}_{2}$; LDM-S $\mathrm{I}_{16}$ (Figure 8L); MG-Pic 10 (Figure 8H); MG-Pic 17 (Figure 8D): isolated footprints (Oliva et al., 2013; Oliva and Arregui, 2016). Study material. (referred to Lamaichnum tulipensis). Pehuen Co; PGT-VII: trackway of twenty-one footprints (holotype) (Figure 7D) (Aramayo and Manera de Bianco, 1987b); PCT-VIII: trackway of four footprints (neotype) (Figure 7F) (Aramayo et al., 2015); MD-YPI-06-04: isolated footprint, in sedimentary block (paratype) (Aramayo et al., 2015); PCT-IX: trackway of thirteen footprints (figures 7E, 8M) and MD-YPI-06-10: silicone rubber cast of this specimen (Aramayo et al., 2015). Laguna del Monte; LDM-S $\mathrm{T}_{1}$ and LDM- $\mathrm{S}_{1} \mathrm{~T}_{2}$ 

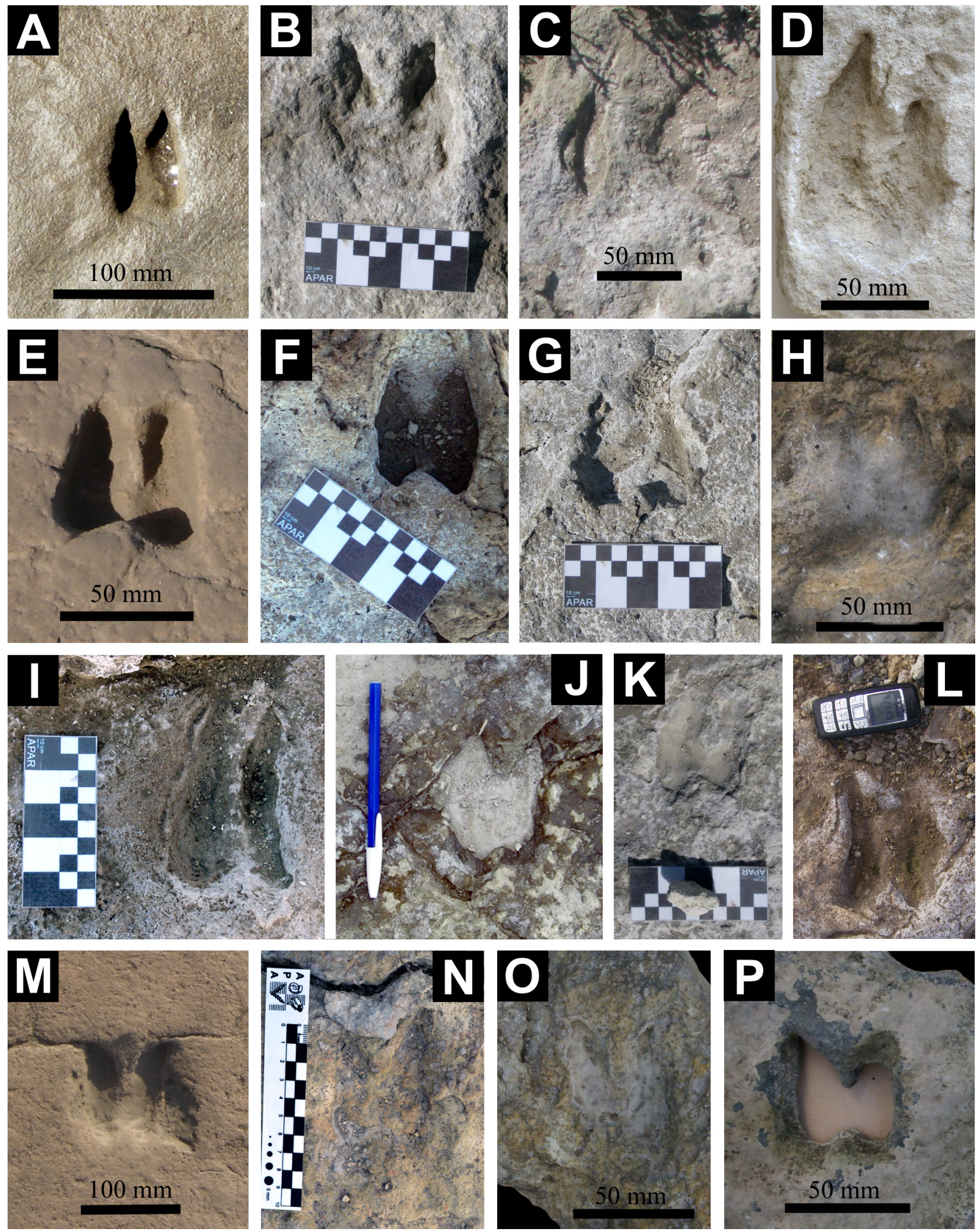

Figure 8 Llamoid paleoichnites (Lamaichnum guanicoe Aramayo and Manera de Bianco, 1987a). Unaltered footprints (healthy feet): A-D: shallowest/lesser-preserved and E-H: deepest/better-preserved, specimens. Altered footprints (unhealthy feet): evidencing I-L: inward/ subparallel and M-P: outward/divergent, toenail overgrowths. Scale bar in B, F, G, I, K, and N equals $10 \mathrm{~cm}$. 
Table 2. Footprint measurements of South American, Late Pleistocene, camelid-trackways: Lamaichnum guanicoe Aramayo and Manera de Bianco, 1987b = Lamaichnum tulipensis (Aramayo and Manera de Bianco, 1987b). L: track length; W: track width; L/W: length/width ratio.

\begin{tabular}{|c|c|c|c|c|c|c|c|c|c|}
\hline \multirow{2}{*}{ Ichnotaxon } & \multirow{2}{*}{ Ichnopathology } & \multirow{2}{*}{\multicolumn{2}{|c|}{ Material }} & Referred & \multicolumn{3}{|c|}{ Measurement Range (mm) } & \multirow{2}{*}{$\mathbf{N}^{\circ}$} & \multirow{2}{*}{ Reference } \\
\hline & & & & Footprint & $\mathbf{L}$ & $\mathbf{W}$ & $\mathbf{L} / \mathbf{W}$ & & \\
\hline \multirow{8}{*}{$\begin{array}{l}\text { Lamaichnum guanicoe } \\
\text { (Aramayo and Manera de } \\
\text { Bianco, 1987b) }\end{array}$} & \multirow{8}{*}{$\begin{array}{l}\text { Unaltered Footrpints } \\
\text { Neither disease } \\
\text { (healthy foot) }\end{array}$} & \multicolumn{2}{|c|}{ Holotype } & Undetermined & $90-100$ & $70-75$ & - & 11 & $\begin{array}{c}\text { Aramayo and Manera } \\
\text { de Bianco, 1987a }\end{array}$ \\
\hline & & \multicolumn{2}{|c|}{ Neotype (MD-YPI-05-15) } & Undetermined & $75-116$ & $46-87$ & $0.94-1.88$ & 21 & $\begin{array}{c}\text { Aramayo et al., 2015; } \\
\text { tab. } 3\end{array}$ \\
\hline & & \multirow{6}{*}{$\begin{array}{c}\text { Hypodigm } \\
\text { (MD-YPI-15-01) }\end{array}$} & I & Undetermined & $90-120$ & $65-80$ & $1.13-1.69$ & 12 & \multirow{6}{*}{ Aramayo et al. , 2015} \\
\hline & & & II & Undetermined & $90-95$ & $65-80$ & $1.19-1.39$ & 4 & \\
\hline & & & III & Undetermined & $90-95$ & $55-80$ & $1.13-1.72$ & 6 & \\
\hline & & & IV & Undetermined & $85-100$ & $65-80$ & $1.14-1.54$ & 15 & \\
\hline & & & V & Undetermined & $85-95$ & $60-85$ & $1.13-1.58$ & 3 & \\
\hline & & & VI & Undetermined & $80-115$ & $60-85$ & $1.14-1.64$ & 9 & \\
\hline \multirow{10}{*}{$\begin{array}{l}\text { Lamaichnum tulipensis } \\
\text { (Aramayo and Manera de } \\
\text { Bianco, 1987b) }\end{array}$} & \multirow{10}{*}{$\begin{array}{l}\text { Altered Footprints } \\
\text { Outward / divergent } \\
\text { toenail overgrowth }\end{array}$} & \multicolumn{2}{|c|}{ Holotype } & Undetermined & $110-120$ & $85-90$ & - & 21 & $\begin{array}{l}\text { Aramayo and Manera } \\
\text { de Bianco, } 1987 \mathrm{~b}\end{array}$ \\
\hline & & \multirow{3}{*}{\multicolumn{2}{|c|}{ Neotype }} & Forefoot & $135-145$ & 100 & $1.35-1.45$ & \multirow{3}{*}{4} & \multirow{6}{*}{$\begin{array}{c}\text { Aramayo et al. }, 2015 ; \\
\text { tab.4 }\end{array}$} \\
\hline & & & & Hindfoot & $100-120$ & $80-92.5$ & $1.25-1.33$ & & \\
\hline & & & & Ichnospecifical & $100-145$ & $80-100$ & $1.25-1.45$ & & \\
\hline & & \multirow{3}{*}{\multicolumn{2}{|c|}{$\begin{array}{c}\text { Hypodigm } \\
\text { (MD-YPI-06-10) }\end{array}$}} & Forefoot & $95-120$ & $70-105$ & $0.90-1.57$ & \multirow{3}{*}{13} & \\
\hline & & & & Hindfoot & $90-110$ & $70-95$ & $1.05-1.28$ & & \\
\hline & & & & Ichnospecifical & $90-120$ & $70-105$ & $0.90-1.57$ & & \\
\hline & & \multirow{2}{*}{\multicolumn{2}{|c|}{ LDM- $\mathrm{S}_{1} \mathrm{~T}_{1}$}} & Forefoot & 120 & $90-105$ & 1.14 & 9 & \multirow{3}{*}{ New Study Material } \\
\hline & & & & Hindfoot & 115 & $85-90$ & $1.27-1.34$ & & \\
\hline & & \multicolumn{2}{|c|}{ LDM-S $\mathrm{T}_{2}$} & Undetermined & $120-125$ & $75-100$ & $1.20-1.67$ & 4 & \\
\hline
\end{tabular}

(Figure 7H): trackways composed of nine and four footprints, respectively; LDM-S $\mathrm{I}_{72} ; \mathrm{LDM}-\mathrm{S}_{2} \mathrm{I}_{7}$; $\mathrm{LDM}_{-} \mathrm{S}_{2}$ (Figure 8N); MG-Pic 11 (Figure 8O); MG-Pic 16 (Figure 8P): isolated footprints (Oliva et al., 2013; Oliva and Arregui, 2016).

Extended diagnosis. (modified from Aramayo and Manera de Bianco, 1987b). Paraxonic didactyl footprints, of digitigrade type, related to small to great-sized artiodactyls of the Camelidae Family. Almost indistinguishable, heart-shaped to subrectangular-subtrapezoidal outlined, forefoot and hindfoot imprints (strong homopody, with a secondary teratologic heteropody). Each ichnite is typically elongated (length/width ratio higher than 1), with bicuspidate anterior margin, bilobed/biconvex posterior border and softly curved lateral walls. Two oblong, acicular to lanceolate, digital impressions (attributed to the prints generated by the toe cushions of digits III-IV); both are closely resembling in shape and size, having broad, rounded posterior edges, narrow acuminate anterior tips, and usually ending in small sharp nail prints; these latter are commonly rectilinear, anteriorly directed, and coincident with the digital axes (stretching and departing from their natural position, either curving inwardly and/or outwardly, in pathological cases of horny overdevelopment). Interdigital septum of contrasting development (depending on the preservational state), expanding forward in a long V-shaped interclavular gap (more or less extended and open, in function of the separation and divergence reached by the digits; distally overexpanded during toenail overgrowth) and rearward in a short parabolic heel notch, of variable amplitude and depth. Interphalangeal trough and medial pocket reduced or absent.

Geographic and chronostratigraphic distribution. Neogene of North America (Lucas and Hunt, 2007; Cabral-Perdomo et al., 2018). 
Table 3. Footprint measurements of South American, Late Pleistocene, camelid-tracks: Lamaichnum guanicoe Aramayo and Manera de Bianco, 1987b = Lamaichnum tulipensis (Aramayo and Manera de Bianco, 1987b). L: track length; W: track width; L/W: length/width ratio.

\begin{tabular}{|c|c|c|c|c|}
\hline \multirow{2}{*}{$\begin{array}{c}\text { Ichnotaxon } \\
\text { (ichnogenus and ichnospecies) }\end{array}$} & \multirow{2}{*}{$\begin{array}{l}\text { Designation } \\
\text { (acronym) }\end{array}$} & \multicolumn{3}{|c|}{ Measurements (mm) } \\
\hline & & $\mathrm{L}$ & $\mathrm{W}$ & $\mathrm{L} / \mathrm{W}$ \\
\hline \multirow{26}{*}{$\begin{array}{c}\text { Unaltered Footprints (Healthy } \\
\text { Feet) } \quad \text { Lamaichnum } \\
\text { guanicoe (Aramayo and Manera } \\
\text { de Bianco, 1987b) }\end{array}$} & LDM-S $\mathrm{I}_{24}$ & 75 & 65 & 1.15 \\
\hline & LDM-S $\mathrm{S}_{1} \mathrm{I}_{25}$ & 65 & 60 & 1.08 \\
\hline & LDM-S $\mathrm{I}_{40}$ & 80 & 65 & 1.23 \\
\hline & LDM-S $\mathrm{I}_{41}$ & 100 & 70 & 1.43 \\
\hline & LDM- $\mathrm{S}_{1} \mathrm{I}_{42}$ & 95 & 75 & 1.27 \\
\hline & LDM-S $\mathrm{I}_{43}$ & 100 & 75 & 1.33 \\
\hline & LDM- $\mathrm{S}_{1} \mathrm{I}_{44}$ & 100 & 75 & 1.33 \\
\hline & LDM-S $\mathrm{I}_{45}$ & 110 & 75 & 1.47 \\
\hline & LDM-S $I_{52}$ & 110 & 70 & 1.57 \\
\hline & LDM-S $\mathrm{S}_{1} \mathrm{I}_{73}$ & 85 & 82 & 1.03 \\
\hline & LDM-S $\mathrm{I}_{78}$ & 75 & 55 & 1.36 \\
\hline & LDM-S $\mathrm{I}_{79}$ & 65 & 50 & 1.3 \\
\hline & LDM- $\mathrm{S}_{1} \mathrm{I}_{82}$ & 100 & 85 & 1.17 \\
\hline & LDM-S $\mathrm{S}_{1} \mathrm{I}_{94}$ & 90 & 70 & 1.2 \\
\hline & LDM-S $\mathrm{I}_{127}$ & 75 & 65 & 1.15 \\
\hline & LDM-S $\mathrm{I}_{128}$ & 95 & 75 & 1.27 \\
\hline & LDM-S $\mathrm{I}_{132}$ & 95 & 65 & 1.46 \\
\hline & LDM-S $\mathrm{I}_{134}$ & 85 & 55 & 1.54 \\
\hline & LDM-S $\mathrm{I}_{176}$ & 100 & 75 & 1.33 \\
\hline & LDM-S $\mathrm{I}_{178}$ & 80 & 65 & 1.23 \\
\hline & LDM-S $\mathrm{I}_{179}$ & 80 & 70 & 1.14 \\
\hline & LDM-S $\mathrm{I}_{5}$ & 105 & 75 & 1.4 \\
\hline & LDM- $\mathrm{S}_{2} \mathrm{I}_{12}$ & 100 & 65 & 1.54 \\
\hline & MG-Pic 10 & 75 & 60 & 1.25 \\
\hline & MG-Pic 17 & 100 & 65 & 1.53 \\
\hline & Population Range & $65-110$ & $50-85$ & $1.03-1.57$ \\
\hline \multirow{5}{*}{$\begin{array}{l}\text { Footprints associated to } \\
\text { Inward / Subparallel } \\
\text { Toenail Overgrowth }\end{array}$} & LDM-S $\mathrm{I}_{180}$ & 120 & 75 & 1.6 \\
\hline & LDM-S $\mathrm{I}_{181}$ & 75 & 60 & 1.25 \\
\hline & $\mathrm{LDM}^{-\mathrm{S}_{2} \mathrm{I}_{6}}$ & 85 & 75 & 1.13 \\
\hline & LDM- $\mathrm{S}_{2} \mathrm{I}_{16}$ & 125 & 110 & 1.13 \\
\hline & Population Range & $75-125$ & $60-110$ & $1.13-1.6$ \\
\hline \multirow{4}{*}{$\begin{array}{l}\text { Footprints associated to } \\
\text { Outward / Divergent } \\
\text { Toenail Overgrowth }\end{array}$} & LDM-S $\mathrm{I}_{72}$ & 125 & 75 & 1.67 \\
\hline & LDM-S $\mathrm{S}_{7}$ & 130 & 80 & 1.63 \\
\hline & LDM-S ${ }_{2} I_{9}$ & 75 & 65 & 1.15 \\
\hline & MG-Pic 11 & 75 & 70 & 1.07 \\
\hline \multirow{2}{*}{$\begin{array}{c}\text { Lamaichnum tulipensis } \\
\text { (Aramayo and Manera de Bianco, } \\
\text { 1987b) }\end{array}$} & MG-Pic 16 & 65 & 60 & 1.08 \\
\hline & Population Range & $65-130$ & $60-80$ & $1.07-1.67$ \\
\hline \multicolumn{2}{|c|}{ Ichnospecifical Range* } & $40-150$ & $50-110$ & $1.03-1.67$ \\
\hline
\end{tabular}

"Extended from: Aramayo and Manera de Bianco, 1987b, 1996; Aramayo et al., 2015; Lucas and Hunt, 2007. 
Late Pleistocene of South America (Aramayo and Manera de Bianco, 1987b, 1996; Tassara et al., 2007; Oliva et al., 2013; Aramayo et al., 2015).

Possible trackmakers. Vicugna vicugna (Molina, 1782) (= Lama gracilis Gervais and Ameghino, 1880) (small size); Lama guanicoe (Müller, 1776) (medium size); $\dagger$ Eulamaops parallelus (Ameghino, 1884) and $\dagger$ Hemiauchenia paradoxa Gervais and Ameghino, 1880 (great size) (Artiodactyla Owen, 1848; Camelidae Gray, 1821; Camelinae Zittel, 1893; Lamini Webb, 1965).

Description. The paleoichnites associated with South American camelids (Lamaichnum guanicoe) are morphostructurally indistinguishable from those generated by living llamoid species, although exhibiting a higher measurement range: $40-150$ mm (see Lucas and Hunt, 2007), revealing the existence of larger extinct forms.

Similar to modern tracks, their appearance is extremely changeable; they display a broad range of extramorphological variations (see Lucas and Hunt, 2007), which depend on autopode-substrate interaction (taphonomical variations) instead of intraspecific and/or interespecific/intergeneric autopodial differences (insignificant in these ungulates).

Consequently, the track morphology would respond to the pressure that the foot exerts over the ground surface, whose intensity varies according to the gait and body mass, and the grade of sediment plasticity, closely linked to factors such as the composition, granulometry, degrees of consolidation, compaction and cementation, moisture content and water saturation level, development of microbial mat, etc.

Unaltered footprints. The imprints produced by healthy feet are almost bisymmetric, typically longer than wide (length/width ratio higher than 1), with length ranging from 65 to $120 \mathrm{~mm}$ and width varying from 45 to $90 \mathrm{~mm}$ (tables 2, 3).

In this sense, forefoot and hindfoot tracks are nearly identical, both morphologically and dimensionally, making their differentiation virtually impossible (strong homopody), even in well-preserved trackways (Figure 7A-C).
These footprints consist of two acuminate, subparallel, digital impressions, which are invariably undivided and uniformly concave, indicating the presence of smooth, antero-posteriorly elongated, toe cushions.

In shallowest and less-preserved specimens, these toepad imprints constitute narrow acicular depressions that remain disconnected through a thick median sulcus (interdigital septum) (Figure 8A-D). This subtype, which does not faithfully reflect the palmar/plantar anatomy of the trackmaker, is typically related to compact sediments.

Meanwhile, in the deepest and better-preserved ichnites, the toepad imprints represent anteriorly pointed and posteriorly rounded lanceolate concavities separated by a reduced medium ridge (which may be occasionally absent) (Figure $8 \mathrm{E}-\mathrm{H}$ ). This subtype, generally linked to uncompacted deposits, is the one that best manifests the palmar/ plantar physiognomy of the producer organism.

In both cases, toenail prints can be present, appearing either as thin lineations and/or sharp/ truncated appendices, which are straight, forwardly directed and coincident with the rectilinear digital axes.

Pathological footprints. Llamoid paleoichnites, exhibiting signs of horny overdevelopment (Figure 7D-H), are relatively frequent in the fossil record. Those attributed to an inward/subparallel clavular overgrowth (Figure 8I-L), while retaining many of the intrinsic features observed in the healthy footprints (e.g., similar track length and track width; parallelism and alignment of toepad- and toenail-prints; regular development of interdigital septum and interclavular spacing), lose their bilateral symmetry and acquire a series of secondary features related to this condition: (1) inward curving of digital axes and digital impressions; (2) elongation and antero-medial projection of toenails (usually accompanied with distal truncation and/or cracking); and (3) uneven sizing of toepad prints, with inner toe (III) comparatively shorter, narrower, and deeper than outer digit (IV).

The paleotracks associated with outward/divergent toenail overgrowth (Figure $8 \mathrm{~N}-\mathrm{P}$ ) depart 
notably from their natural morphology, conserving a bilateral symmetry, although undergoing deep anatomical modifications: (1) forwardly divergent, outwardly facing, digital impressions, which, despite maintaining a normal development of the toepad imprints, experienced hypertrophy and lateral bending of the toenail prints; (2) distal overexpansion of the interclavular gap, with anomalous separation between both digital ends and curving of the axial borders; (3) longitudinal enlargement and transversal widening of the tracks, due to the antero-lateral projection of the overgrown nails; (4) normal acquisition of a secondary heteropody, owing to a differential upgrowth of manual and pedal toenails, greater in the first ones; (5) deeper imprinted footprints, because the higher pressure exercised by the affected limb, being specially notable this condition in the forefeet; and (6) the interdigital septum, although retaining a regular development, appears to be shorter than in unaltered footprints due to the anterior projection of the overdeveloped nails.

Ichnotaxonomic remarks. Traditionally, two llamoid ichnospecies are recognized for the Late Pleistocene of South America: Lamaichnum guanicoe and Lamaichnum tulipensis (= Megalamaichnum tulipensis) (Aramayo et al., 2015).

Aramayo and Manera de Bianco (1987b, 1996) segregate initially these ichnotaxa, both at ichnospecific and ichnogeneric level: Lamaichnum guanicoe and Megalamaichnum tulipensis; mainly due to the greater dimensions and digital divergence of the latter.

Lucas and Hunt (2007) doubted the usefulness of such features as potential ichnotaxobases, proposing to synonymize both ichnospecies and consequently the ichnogenera Megalamaichnum with Lamaichnum.

Despite Aramayo et al. (2015) accepting the generic synonymy suggested by these authors, they retained a specific distinction between Lamaichnum guanicoe and Lamaichnum tulipensis nov. comb., based upon the heteropody exhibited by the latter ichnospecies, with well-differentiated forefoot and hindfoot imprints: the first ones slightly larger and deeper impressed, showing pointed anterior ends facing outward, while the second ones culminating in subparallel, forwardly directed, digital tips.

Recently, Oliva and Arregui (2016) recognize the same morphological pattern of Megalamaichnum tulipensis in footprints of modern camelids affected by horny overdevelopment and the consequent deformation of the toenails (hypothesis herein analysed and confirmed).

More precisely, the new available evidence indicates that the ichnotaxobases established to differentiate Megalamaichnum tulipensis from Lamaichnum guanicoe (and the ichnogenera that they typify) (see Aramayo and Manera de Bianco, 1987b, 1996; Aramayo et al., 2015) represent signs of outward/divergent toenail overgrowth in living llamoids (falling within the range of intraspecific variation of an unique species) that should not be interpreted as differences of ichnotaxonomical order.

Given the exposed results, we synonymize Lamaichnum guanicoe and Megalamaichnum tulipensis as well as the ichnogenera Lamaichnum and Megalamaichnum, reaffirming the criteria of Lucas and Hunt (2007).

Ichnorder Perissodactypedida Vialov, 1966

Morphofamily Hippipedidae Vialov, 1966 Ichnogenus Hippipeda Vialov, 1966

Type ichnospecies. Hippipeda aurelianis Vialov, 1966.

Hippipeda isp.

(Table 4; Figure 9)

Study material. Pehuen Co; PCT-X: trackway of four footprints, including two tracks and two undertracks (Figure 9C) (Manera de Bianco and Aramayo, 2003; Aramayo et al., 2015); PCT-XI: trackway of four footprints (Figure 9E). Laguna del Monte; $\mathrm{LDM}-\mathrm{S}_{1} \mathrm{~S}_{15}$ (Figure 9I) and LDM-S $\mathrm{S}_{2} \mathrm{~S}_{6}$ (Figure 9A): couples of superimposed forefoot and hindfoot tracks; $\mathrm{LDM}-\mathrm{S}_{2} \mathrm{~S}_{10}$ : three associated 

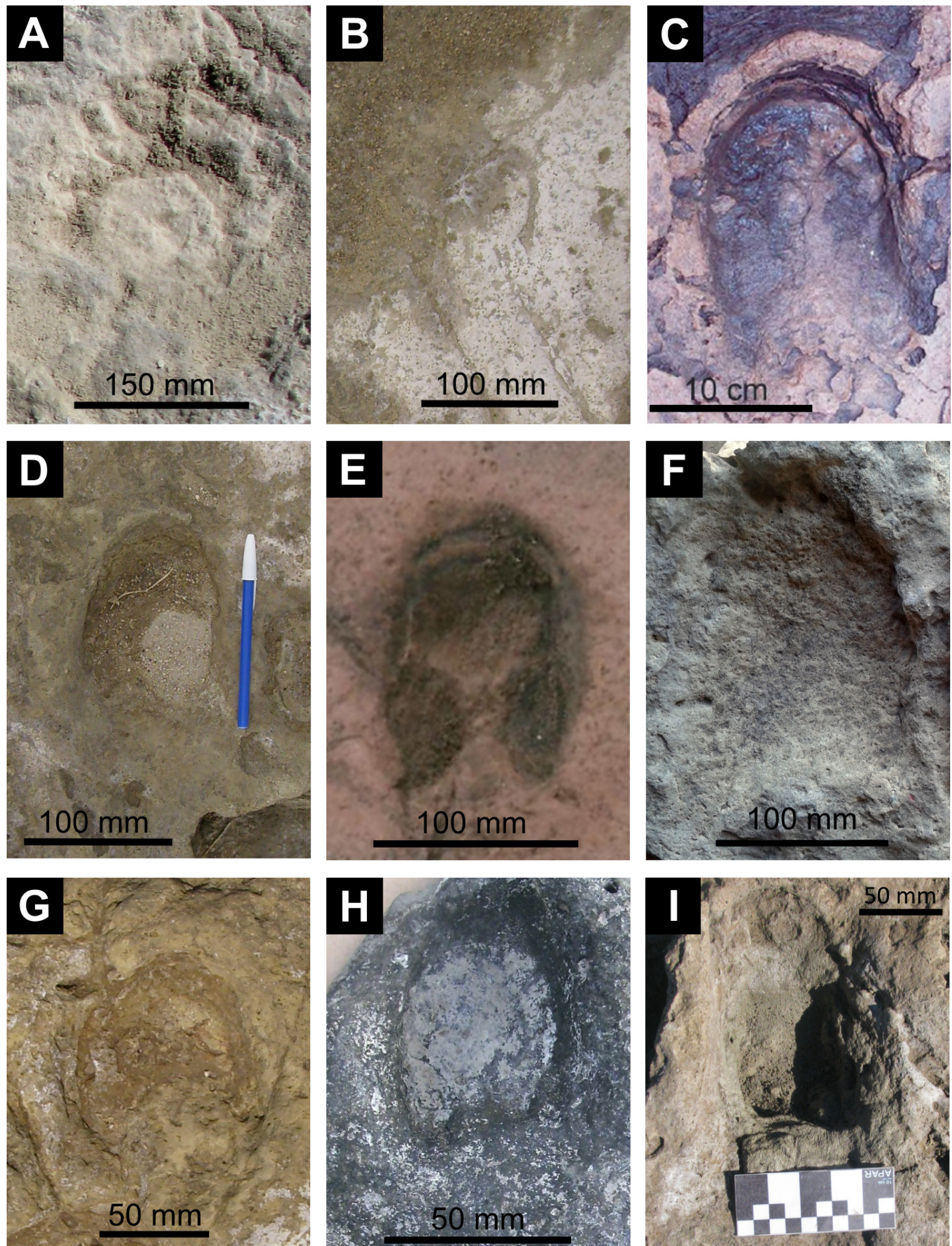

Figure 9 Equid paleoichnites (Hippipeda isp.). Unaltered footprints (balanced hooves): (A-B) forefoot hoofwall imprints; (C-D) natural hindfoot tracks. Altered footprints (unbalanced hooves): (E-F) rearfoot impressions showing potential signs of contracted -heel hooves; (G-H) hindfoot imprints evidencing asymmetrical distortion of the hooves; (I) forefoot track associated with a turkish-slipper foot. C, Taken from Manera-de Bianco and Aramayo (2013). 
footprints (Figure 9G); LDM-S $\mathrm{T}_{4}$ (Figure 9D) and LDM-S $\mathrm{S}_{5}$ (Figure 9B): two related trackways (each one composed of three footprints); MG-Pic 7 (Figure 9F) and MG-Pic 8 (Figure 9H): isolated footprints, preserved in loose slabs (Oliva et al., 2013; Oliva and Arregui, 2016); all specimens remain in situ (excepting MG-Pic 7 and MG-Pic 8).

Diagnosis. Monodactyl mesaxonic footprints, of unguligrade type, related to moderate-sized hoofed mammals of the Equidae Family. Almost undifferentiated forefoot and hindfoot tracks (slight heteropody) consisting of a single hemiellipsoidal to hemiovoidal toe impression (attributed to the trace generated by a central digit III); distinguished basically through their contrasting dimensions and proportions (pes comparatively smaller and narrower than manus). On better-preserved specimens, an excavated and strongly curved hoofwall print, enclosing a more elevated, downward concave solar region, can be observed; on each side, the posterior ends of the wall bend forward and inward in wedge-shaped bar marks. Surrounded by the latter structures, on the posterior half of the track, a subtriangular inner frog print usually appears; limited posterolaterally by two mirrored, heel-bulb impressions separated by a median talonar notch.

Possible trackmakers. Equи (Amerhippus) nеоgeus Lund, 1840 (medium size); Hippidion principale (Lund, 1846) and Hippidion devillei (Gervais, 1855)

Table 4. Footprint measurements of South American, Late Pleistocene, horse-tracks: Hippipeda isp. L: track length; W: track width; H: hoofwall thickness; L/W: length/width ratio.

\begin{tabular}{|c|c|c|c|c|c|c|c|c|c|}
\hline \multirow{2}{*}{\multicolumn{2}{|c|}{ Hoof Typology }} & \multirow{2}{*}{\multicolumn{3}{|c|}{$\begin{array}{l}\text { Designation } \\
\text { (acronym) }\end{array}$}} & \multirow{2}{*}{$\begin{array}{l}\text { Referred } \\
\text { Footprint }\end{array}$} & \multicolumn{4}{|c|}{ Measurements (mm) } \\
\hline & & & & & & $\mathrm{L}$ & W & $\mathrm{H}$ & $\mathrm{L} / \mathrm{W}$ \\
\hline \multirow{13}{*}{\multicolumn{2}{|c|}{$\begin{array}{c}\text { Unaltered = Balanced } \\
\text { Hooves }\end{array}$}} & \multicolumn{3}{|c|}{ MG-Pic 6} & Forefoot & 105 & 85 & 10 & 1.24 \\
\hline & & \multirow{2}{*}{ LDM-S ${ }_{2} \mathrm{~S}$} & & $\mathrm{I}_{1}$ & Forefoot & 150 & 120 & 10 & 1.25 \\
\hline & & & & $\mathrm{I}_{2}$ & Forefoot & - & 115 & 10 & - \\
\hline & & \multirow{3}{*}{\multicolumn{2}{|c|}{ LDM- $S_{2} T_{4}$}} & $\mathrm{I}_{1}$ & Forefoot & 150 & 120 & - & 1.25 \\
\hline & & & & $\mathrm{I}_{2}$ & Hindfoot & 115 & 90 & 8 & 1.27 \\
\hline & & & & $\mathrm{I}_{3}$ & Hindfoot & 120 & 95 & 8 & 1.26 \\
\hline & & \multirow{3}{*}{$\mathrm{LDM}_{-} \mathrm{S}_{2} \mathrm{~T}$} & & $\mathrm{I}_{1}$ & Forefoot & 80 & 70 & 8 & 1.14 \\
\hline & & & & $\mathrm{I}_{2}$ & Forefoot & 80 & 70 & 8 & 1.14 \\
\hline & & & & $\mathrm{I}_{3}$ & Hindfoot & 60 & 50 & 8 & 1.2 \\
\hline & & \multirow{2}{*}{ PCT-X* } & & & Forefoot & 150 & 120 & - & 1.25 \\
\hline & & & & & Hindfoot & 130 & 100 & - & 1.3 \\
\hline & & \multirow{2}{*}{\multicolumn{3}{|c|}{ Dimensional Range }} & Forefoot & $80-150$ & $70-120$ & & $1.14-1.25$ \\
\hline & & & & & Hindfoot & $60-130$ & $50-100$ & & $1.20-1.30$ \\
\hline \multirow{7}{*}{$\begin{array}{c}\text { Altered = } \\
\text { Unbalanced } \\
\text { Hooves }\end{array}$} & Contracted Heel & MG & & & Undetermined & 105 & 65 & 10 & 1.62 \\
\hline & \multirow{4}{*}{ Distorted Hoof } & \multicolumn{3}{|c|}{ MG-Pic 8} & Hindfoot & 85 & 65 & - & 1.3 \\
\hline & & \multirow{3}{*}{\multicolumn{2}{|c|}{$\mathrm{LDM}-\mathrm{S}_{2} \mathrm{~S}_{10}$}} & $\mathrm{I}_{1}$ & Forefoot & 125 & 100 & - & 1.25 \\
\hline & & & & $\mathrm{I}_{2}$ & Hindfoot & 100 & 80 & 10 & 1.25 \\
\hline & & & & $\mathrm{I}_{3}$ & Hindfoot & 105 & 85 & - & 1.23 \\
\hline & \multirow{2}{*}{$\begin{array}{c}\text { Turkish Slipper } \\
\text { Hoof }\end{array}$} & \multirow{2}{*}{\multicolumn{2}{|c|}{$\mathrm{LDM}_{-} \mathrm{S}_{1} \mathrm{~S}_{15}$}} & $\mathrm{I}_{1}$ & Forefoot & 162.5 & 80 & - & 2.03 \\
\hline & & & & $\mathrm{I}_{2}$ & Hindfoot & 157.5 & 90 & - & 1.75 \\
\hline
\end{tabular}


(small size) (Perissodactyla Owen, 1848; Equidae Gray, 1821; Equinae Gray, 1821; Equini Gray, 1821).

Geographic and chronostratigraphic distribution. Late Pleistocene of Buenos Aires Province, Argentina (Manera de Bianco and Aramayo, 2003; Aramayo et al., 2007, 2015; Oliva et al., 2013).

Description. The paleoichnites related to South American equids, from Pehuen Co (Manera de Bianco and Aramayo, 2003; Aramayo et al., 2015), Monte Hermoso (Aramayo et al., 2007) and Laguna del Monte (Oliva et al., 2013) exhibit the monodactyl track-pattern distinctive of the ichnogenus Hippipeda.

They display, on the other hand, the autopodial conformation exhibited by the modern equids, with an expanded ungular region (encircled by a thick hoofwall print) and a forwardly projected frog area.

Unaltered footprints. Those equid tracks reflect a normal anatomy of the producer autopode (balanced hoof), constituting elongate hemi-ellipsoidal impressions, which can be evenly sunk (compact substrate) and/or deeper forward (uncompacted sediment).

Forefoot and rearfoot imprints exhibit the same morphostructural pattern, only differentiating through their dimensions and proportions (slight heteropody). In this regard, the manus (length: $80-150 \mathrm{~mm}$; width: $70-120 \mathrm{~mm}$; length/width ratio: $1.14-1.25)$ is comparatively larger and proportionally longer than the pes (length: $60-130$ $\mathrm{mm}$; width: $50-100 \mathrm{~mm}$; length/width ratio: $1.20-1.30)$ (Table 4).

The hoofwall print constitutes a deep perimetral flange, of epicycloidal (Figure 9A) to U-shaped (Figure 9B) outline, with curvilinear and rectilinear lateral walls (quarters), respectively; a uniformly convex frontal wall (toe); and a nearly constant thickness $(8-10 \mathrm{~mm})$ that determines the circumference of the track.

On each side, this structure defines a V-shaped postero-external angle (heel buttress) and then bends and sharps forwardly and inwardly (antero-medially) in a wedge-shaped bar mark separated from its opposite by the frog apex.

Enclosed inside the hoofwall, a medium crescent-shaped sole region (radially distributed), a subtriangular inner frog print (axially positioned), and two postero-external heel-bulb impressions (symmetrically disposed on both sides of the track axis) may be present sometimes.

Invariably, the balanced hoofprints (unaltered tracks) are characterized by being almost bisymmetric and having transversely aligned heel buttresses, disposed approximately at the same level as the frog baseline and the heel-bulb impressions (Figure 9C-D).

Pathological footprints. Equid paleoichnites, evidencing potential signs of "unbalanced hooves" with contracted heel (Figure 9E-F), conserve the bilateral symmetry and an unaltered hoofprint (ungular area). These differs specifically from healthy footprints by their greater antero-posterior elongation and transversal compression (length/ width ratio $>1.30$ ) (Table 4), as well as by exhibiting a narrowing and distal projection of the talonar region, with an approximation of both heel-bulb impressions and an enlargement of the space existent between the frog baseline and heel buttresses.

The footprints indicating bilateral distortion of the hoof (Figure 9G-H), while maintaining similar dimensions and proportions to the unaltered tracks (length/width ratio $\leq 1.30)$ (Table 4), are distinguished immediately by their asymmetry, clearly reflected either into the hoofwall print or the solar region, through a series of distinctive features characteristic of this anomaly: (1) unequilateral, eccentric (sagitally disproportionate), frontal wall (toe); (2) medial wall (inner quarter) abnormally longer and straighter than the external wall (outer quarter); (3) forwardly projected internal pillar (toe-quarter junction); (4) anteriorly displaced external heel buttress; and (5) uneven development and distribution of sole area (solar asymmetry).

Finally, the fossil tracks attributed to "turkish-slipper feet" (Figure 9I) depart notably from their 
natural morphology, manifesting a significant longitudinal enlargement (length/width ratio $\geq 1.75$ ) (Table 4) with a considerable lengthening (distal projection) of the hoofprint, and an eventual loss of the bilateral symmetry.

Likewise, these paleoichnites display several secondary characteristics linked to the hypertrophy and deformation of the ungular region: (1) plantar imprint (toe impression) unusually deeper rearward due the antero-dorsal bending of the affected hoof; (2) lateral walls (inner and outer quarters) prolonging longitudinally and ending postero-medially in overexpanded subparallel bar marks (unlike those medially convergent of unaffected feet); and (3) overdevelopment and anterior expansion of the frog print (which do not sharpen forward in an acute apex, as occurs in healthy hooves).

Ichnotaxonomic remarks. A comprehensive comparative study on modern equids allows the recognition of three primary factors that are determinant in the specific differentiation of hooves and hoofprints: (1) hoofwall morphology (e.g., height, contour, thickness, inclination, and conicity of the wall); (2) sole sagging (profundity and degree of concavity/flattening); and (3) frog development (antero-posterior elongation and transversal widening).

As previously discussed, most of these features are also subject to intraspecific variations of teratologic order, as well as the overall footprint measurements (length, width, length/width ratio). Therefore, it is advisable to restrict its use only to unaltered hoofprints (non-pathological tracks).

Regardless, the hoofwall profile and curvature of lateral walls (quarters) remain relatively constant on conspecific individuals (either in balanced or unbalanced hooves). So, herein they are proposed as the most reliable diagnostic characters.

In fossil tracks, besides the hoofwall shape (Scrivner, 1984; Scrivner and Bottjer, 1986; Sarjeant and Reynolds, 1999; Santucci and Nyborg, 1999; Santucci et al., 2011), the length/width ratio, hoofprint area, hoofwall thickness, presence/ absence of frog print, and morphology/extension of bar marks (see Reynolds, 2006; McNeil et al., 2007) are particularly relevant in the distinction of ichnospecies.

The austral records of Hippipeda, from the Late Pleistocene of Pampean Region of Argentina (Manera de Bianco and Aramayo, 2003; Aramayo et al., 2007, 2015; Oliva et al., 2013; Oliva and Arregui, 2016), have not been assigned to a specific level.

In this context, the contrasting hoofwall profiles and quarter convexities observed in the study material suggest preliminarily the presence of more than one South American equid ichnospecies. Although, it is necessary to deepen the ichnotaxonomic analysis in order to differentiate and define correctly each one of them.

\section{Discussion}

When it comes to vertebrate ichnotaxonomy, it is imperative that the diagnoses of the ichnotaxa are based strictly on morphostructural criteria (excluding all kinds of secondary deviations unlinked to the pedal physiognomy). So, it is always recommended to work with the best-preserved footprints, choosing carefully ichnotaxobases which only reflect anatomical characteristics of the producer foot (Lucas, 2001, 2005).

Nevertheless, not all physical features of a track or trackway are applicable as diagnostic and/or differential criteria. In this regard, the most useful characterictics are those intrinsically related to the structure and functionality of the autopodes, while the "intraspecifical or intramorphological variations" such as ontogenetic changes, sexual dimorphism, pathological and/or congenital anomalies, necessarily lack taxonomic validity.

Then it is necessary to identify this type of intrataxonomic alterations in paleotracks, as far as possible, through comparative analysis with traces 
of modern species. Accordingly, from an ichnopathological viewpoint, it is fundamental to detect clinical signs (related to limb affections known in modern vertebrates) that permit the recognition of pathologic footprints. Consequently, the comparison with living members of the treated groups (a standard procedure used in paleopathology, see Rothschild and Martin, 1993, 2006) becomes specifically important.

Additionally, it is expected that a limb affected by some type of podal disease somehow alters its shape or operation, producing anomalous tracks and trackways that reflect this condition, so that the pathologies develop particular and repetitive ichnological patterns that allow their correct identification (Rothschild and Martin, 2006; McCrea et al., 2014, 2015).

Certain limb affections, such as exungulation, missing digit, or osseous and horny lesions (e.g., injuries, trauma, fissures, and fractures), show a rather erratic distribution, occurring isolatedly and/or differentially (not affecting all extremities). The solar cracking and hornwall fissuration, herein described for proboscidean paleoichnites (Proboscipeda australis), constitute examples of these maladies.

Hence, in sets and trackways combining healthy and unhealthy footprints, it is crucial to restrict the systematic diagnosis of an ichnotaxon to the unaltered elements (unaffected feet) (e.g., McCrea et al., 2014).

In contrast, other podal disorders (e.g., polydactyly, horny overdevelopment, hooves distortion and deformation, laminitis) tend to affect more proportionately to the limbs, commonly preserving the mirrored symmetry and the specular anatomy of the opposite autopodes (at least considering separately fore- and hind-feet).

In these cases, characterized by a greater regularity of the resulting trackways, it is much more difficult to distinguish successfully altered from unaltered imprints. The ichnopathologic research of llamoid
(Lamaichnum guanicoe) and equid (Hippipeda isp.) fossil tracks expound clearly this problematic.

McCrea et al. (2015) proposed five categories of primary factors causative of pathologies, with the potential of affecting firstly the foot morphology, and ultimately the locomotory function of an animal: (1) congenital abnormalities, (2) biomechanical injuries, (3) brain or spinal lesions, (4) sicknesses, and (5) age-related problems. The described diseases would be caused by the fourth group.

The formation of an ichnite is the result of a complex interaction between the autopode and the substrate. The track morphology is not only determined by the podal anatomy, but also depends on extrinsic agents such as the trackmaker behavior (e.g., foot pressure, speed, motion, gait type), the nature of the track-bearer deposits (e.g., composition, granulometry, moisture content, degree of consistency, infaunal activity), and properties of the fluid/sediment interface (e.g., subaerial and/or subaquatic environment, flow energy, weathering intensity, sedimentation and erosion rates, presence of superficial microbial mat, development of a vegetal cover) (Minter et al., 2007; Falkingham, 2014; Falkingham et al., 2014; McCrea et al., 2015).

Therefore, there is a great number of external factors, either syn- or post-depositional (Schulp and Brokx, 1999; Schulp, 2002), as well as a series of pre- and post-impressional effects (McCrea et al., 2015), that can actively influence the footprint appearance and trackway quality, and that must be properly recognized and systematically excluded from the diagnosis of the ichnotaxon.

The ichnological features that are products of any alteration that does not reflect the podal constitution of the trackmaker receive the denomination of "extramorphological characters" (Peabody, 1948), while the ichnotaxa erected from these characteristics are called "phantom taxa" (Haubold, 1996). Within this main category, a subclass of ichnotaxa 
termed "taphotaxa" is distinguished, based strictly on taphonomical induced characters (Lucas, 2001)

Necessarily, phantom taxa and taphotaxa should be considered invalid, because they are diagnosed through non-anatomical ichnotaxobases.

Based on the exposed, we define the intraspecific variations linked to an unhealthy condition of the trackmaker as "ichnopathological characters", which constitute intramorphological secondary features that reflect faithfully the podal anatomy (in this case unhealthy autopodes), differentiating from those extramorphological, due to extrinsic factors, that usually depart from the real foot physiognomy.

On the other hand, we denominate "teratotaxa" to those ichnotaxa based on ichnopathological characters, that constituting "teratologic synonyms" of previously known ichnospecies.

\section{Conclusions}

The analysis of paleoichnites and trackways, corresponding to Holarctic ungulates from the Late Pleistocene (Lujanian Stage/Age) of Pehuen Co and Laguna del Monte tracksites (Buenos Aires Province, Argentina), allows the recognition of several clinical signs that reflect some of the main pedal affections and alterations experienced by living members of the group:

- Proboscidean tracks (Proboscipeda australis) present occasional fissures on the hoofprints (interpreted as vertical fractures on the hornwall and transversal cracking of the sole), which represent significant paleopathology evidence, but simultaneously lacks of direct ichnotaxonomic implications.

- Equid footprints (Hippipeda isp.) exhibit a series of morphological anomalies of the digital impression, linked to contracted heel, distorted hoof, and turkish-slipper foot (three habitual podal disorders in modern horses). The future recognition and study of these intraspecific variations will be essential to achieve a correct ichnosystematic interpretation and definition of the ichnotaxon (both at ichnospecific and ichnogeneric level).

- Lamoid footprints (Lamaichnum guanicoe) display strong evidence of overgrown horn (the most frequent claw disorder in extant camelids), which are often utilized to segregate a second ichnospecies: Lamaichnum tulipensis (= Megalamaichnum tulipensis) (see Aramayo and Manera de Bianco, 1987b, 1996; Aramayo et al., 2015). However, these morphological changes clearly fall within the range of intraspecific variation of a unique species and should not be interpreted as differences of ichnotaxonomical order. Therefore, we propose to synonymize both ichnospecies, and the ichnogenera that these typify, reaffirming the criteria of Lucas and Hunt (2007).

The evidence exposed in this study clarify the need to establish ichnotaxonomic schemes in tetrapods only based on morphostructural characters, excluding secondary intramorphological (e.g., ontogenetic, dimorfic, pathologic) and extramorphological (e.g., taphonomic, postural, locomotional) variations.

\section{Acknowledgements}

This work was supported by Municipio de Guaminí, Dirección Provincial de Museos y Preservación Patrimonial (Secretaría de Cultura de la Provincia de Buenos Aires) and Y-Tec/CONICET. We are grateful to the directives and the staff of "Marcelino Freyre" (Guaminí) and "Carlos Darwin" (Punta Alta) Museums, for allowing us to access their respective paleoichnologic collections. We also want to thank the reviews, comments and suggestions made by the arbiters (Dr. Richard McCrea and Dr. Bruce Rothschild), and the editors (Elizabeth Chávez and Tom Musselman), who improved substantially this paper. We specially dedicate this contribution to Ph. Silvia Aramayo and Dr. Teresa Manera, whose pioneer studies in paleoichnology 
of Late Pleistocene Bonaerian vertebrates contributed initially to the recognition and understanding of the analyzed ichnotaxa.

\section{References}

Abel, O., 1935, Vorzeitliche Lebensspuren: Jena, Germany, Gustav Fisher Verlag, 644 p.

Ameghino, F., 1884, Excursiones geológicas y paleontológicas en la Provincia de Buenos Aires: Boletín de la Academia Nacional de Ciencias en Córdoba, Républica Argentina, $6,161-257$.

Ameghino, F., 1888, Rápidas diagnosis de algunos mamíferos fósiles nuevos de la República Argentina. Obras Completas: Buenos Aires, Argentina, Pablo E. Coni e hijos, 5, 469-480.

Aramayo, S., 2006, Procesos tafonómicos y paleopatológicos observados en un ejemplar de Panochthus tuberculatus (Owen) (Xenarthra, Glyptodontidae) (Pleistoceno tardío) Quequén Salado, Provincia de Buenos Aires, Argentina, in Actas III Congreso Argentino de Cuaternario y Geomorfología: Córdoba, Argentina, Agencia Córdoba Ciencia S.E., 737-741.

Aramayo, S., Manera de Bianco, T., 1987a, Hallazgo de una icnofauna continental (Pleistoceno tardío) en la localidad de Pehuen Go (Partido de Coronel Rosales) Provincia de Buenos Aires, Argentina. Parte I: Edentata, Litopterna, Proboscidea, in Suárez-Riglos, M., Suárez-Soruco, R. (eds.), Actas IV Congreso Latinoamericano de Paleontología: Santa Cruz de la Sierra, Bolivia, 1, 516-531. Aramayo, S., Manera de Bianco, T., 1987b, Hallazgo de una icnofauna continental (Pleistoceno tardío) en la localidad de Pehuen
Co (Partido de Coronel Rosales) Provincia de Buenos Aires, Argentina. Parte II: Carnivora, Artiodactyla y Aves, in Suárez-Riglos, M., Suárez-Soruco, R. (eds.), Actas IV Congreso Latinoamericano de Paleontología: Santa Cruz de la Sierra, Bolivia, 1, 532-547.

Aramayo, S., Manera de Bianco, T., 1996, Edad y nuevos hallazgos de icnitas de mamíferos y aves en el yacimiento paleoicnológico de Pehuen Co (Pleistoceno tardío), Provincia de Buenos Aires, Argentina: Publicación Electrónica de la Asociación Paleontológica Argentina, 4(1), 47-57.

Aramayo, S., Manera de Bianco, T., 2000, Primer hallazgo de icnitas de mamíferos pleistocenos en "Playa del Barco", Pehuen Co, Provincia de Buenos Aires, Argentina: Ameghiniana Suplemento Resúmenes, 37(4), 19R.

Aramayo, S., Di Martino, V., Sánchez, N., 2007, Nuevos registros de huellas de mamíferos pleistocenos en la localidad de Monte Hermoso, Provincia de Buenos Aires, Argentina, in XXIII Jornadas Argentinas de Paleontología de Vertebrados, Libro de resúmenes: Trelew, Argentina, 2-3.

Aramayo, S., Manera de Bianco, T., Bastianelli, N., Melchor, R., 2015, Pehuen Co: Updated taxonomic review of a late Pleistocene ichnological site in Argentina: Palaeogeography, Palaeoclimatology, Palaeoecology, 439, 144-165.

Aufderheide, A., Rodriguez-Martin, C., 1998, The Cambridge Encyclopedia of Human Paleopathology: Cambridge, England, Cambridge University Press, 71-82.

Avanzini, M., Pinuela, L., Garcia-Ramos, J., 2008, Theropod palaeopathology inferred from a Late Jurassic trackway, Asturias (N. Spain): Oryctos, 8, 71-75. 
Baker, J., Brothwell, D., 1980, Animal Diseases in Archaeology: London, England, Academic Press, 235 p.

Barbosa, F., Luna, C., 2014, Lesiones en la coraza y endoesqueleto de un ejemplar de Panochthus Burmeister, 1866 (Mammalia, Glyptodontia) de la Provincia de Córdoba, Argentina: Revista del Museo Provincial de Ciencias Naturales "Dr. Arturo Umberto Illia", 1, 9-26.

Barbosa, F., Porpino, K., Fragoso, A., dos Santos, M., 2013, Osteomyelitis in Quaternary mammal from the Rio Grande do Norte State, Brazil: Quaternary International, 299, 90-93.

Barbosa, F., Porpino, K., Fragoso, A., Oliveira, E., 2014a, Arthritis in a glyptodont (Mammalia, Xenarthra, Cingulata): PLOS One, 9(2), e88646.

Barbosa, F., Araújo, H., Oliveira, E., 2014b, Neck osteoarthritis in Eremotherium laurillardi (Lund, 1842; Mammalia, Xenarthra) from the Late Pleistocene of Brazil: International Journal of Paleopathology, 6, 60-63.

Barbosa, F., Araújo, H., Mothé, D., dos Santos, L., 2017a, Osteological diseases in an extinct Notiomastodon (Mammalia, Proboscidea) population from the Late Pleistocene of Brazil: Quaternary International, 443, 228-232.

Barbosa, F., Porpino, K., Bergqvist, L., Rothschild, B.M., 2017b, Elucidating bone diseases in brazilian pleistocene sloths (Xenarthra, Pilosa, Folivora): first cases reported for the Nothrotheriidae and Megalonychidae Families: Ameghiniana, 54(3), 331-340.

Bartosiewicz, L., 2008, Taphonomy and palaeopathology in archaeozoology: Geobios, 41(1), 69-77.

Bell, P., 2010, Palaeopathological changes in a population of Albertosaurus sarcophagus from the Upper Cretaceous Horseshoe Canyon
Formation of Alberta, Canada: Canadian Journal of Earth Sciences, 47(9), 1263-1268. Bell, P., Rothschild, B., Tanke, D., 201 1, First report of gout in an ornithomimid (Dinosauria, Theropoda) from the Horseshoe Canyon Formation, Alberta, in Sullivan, R., Lucas, S., Spielmann, J. (eds.), Fossil record 3: Albuquerque, U.S.A., New Mexico Museum of Natural History and Science Bulletin, 53, $166-168$

Bennett, S., 2003, A survey of pathologies of large pterodactyloid pterosaurs: Palaeontology, 46(1), 185-198.

Benz, A., 2005, La uña del elefante: morfología macroscópica y microscópica considerando sus cambios patológicos: Zürich, Switzerland, University of Zürich, Institute of Veterinary Anatomy, $\mathrm{PhD}$ thesis, $145 \mathrm{p}$.

Blumenbach, J., 1797, Abbildungen naturhistorischer Gegenstände: Göttingen, Germany, Dieterich, 2 (11-20), 1-22.

Boddaert, P., 1785, Elenchus animalium, volumen 1: Sistens quadrupedia huc usque nota, erorumque varietates: Rotterdam, Netherlands, G.R. Hake, 174 p.

Burnett, G., 1829, Illustrations of the Quadrupeda, or quadrupeds, being the arrangement of the true four-footed beasts, indicated in outline: QuarterlyJournal of Science, Literature, and Art, 336-353.

Cabral-Perdomo, M.A., Bravo-Cuevas, V.M., Pérez-Pérez, A., García-Cabrera, N., 2018, Descripción de las huellas de camélidos y félidos de la localidad Pie de Vaca, Cenozoico Tardío de Puebla, centro de México y algunas consideraciones paleobiológicas: Boletín de la Sociedad Geológica Mexicana, 70(2), 397-416.

Cabrera,A., 1929, Una revisión de los Mastodontes Argentinos: Revista del Museo de La Plata, $32,61-144$. 
Currie, P., Badamgarav, D., Koppelhus, E., 2003, The first Late Cretaceous footprints from the Nemegt locality in the Gobi of Mongolia: Ichnos, 10(1), 1-13.

Cuvier, G., 1800, Leçons d'Anatomie Comparée: Paris, France, Baudouin, 1, 521 p.

Dantas, P., dos Santos, V., Lockley, M., Meyer, C., 1994, Footprint evidence for limping dinosaurs from the Upper Jurassic of Portugal: Gaia, 10, 43-48.

Druetta, H.S., 2009, Evidencias patológicas en un ejemplar de gliptodonte del Pleistoceno de la Provincia de Córdoba, Argentina in III Congreso de la Asociación de Paleopatología en Sudamérica, Libro de Resúmenes: Necochea, Argentina, 86.

Druetta, H.S., 2012, Patologías infecciosas en un ejemplar de Glyptodon Owen (Mammalia, Cingulata, Glyptodontidae) del Pleistoceno de la Provincia de Córdoba, Argentina: Ameghiniana, 49(4) Suplemento Resúmenes, R45-46.

Falkingham, P., 2014, Interpreting ecology and behaviour from the vertebrate fossil track record: Journal of Zoology, 292(4), 222-228.

Falkingham, P., Hage, J., Bäker, M., 2014, Mitigating the Goldilocks effect: the effects of different substrate models on track formation potential: Royal Society Open Science, 1(3), 140225.

Farke, A., O'Connor, P., 2007, Pathology in Majungasaurus crenatissimus (Theropoda: Abelisauridae) from the Late Cretaceous of Madagascar: Journal of Vertebrate Paleontology Memoir, 27, 180-184.

Ferigolo, J., 1992, Non-human vertebrate paleopathology of some Brazilian Pleistocene Mammals, in Araújo, A., Ferreira, L. (eds.), Paleopatologia, Paleoepidemiologia. Estudos multidisciplinares: Rio de Janeiro, Brazil, Escola Nacional de Saúde Pública, Fundação Oswaldo Cruz, 213-234.
Ferigolo,J., 2007, Paleopatologia em Mamíferos, in Carvalho, I., Fernandes, A. (eds.), Icnologia: São Paulo, Sociedade Brasileira de Geologia, 88-107.

Ferreira, D., Ferigolo, J., Ribeiro, A., 2013, Paleopatología em fêmur de Panochthus Burmeister, 1866 (Xenarthra, Cingulata, Glyptodontidae) do Pleistoceno do Estado do Rio Grande Do Sul, Brasil: Paleontologia em Destaque, 66, 60.

Fowler, M., 1978, Hoof problems in zoo animals: Proceedings of the American Association of Zoo Veterinarians, 1978, 88-106.

Fowler, M., 1980, Hoof, claw and nail problems in nondomestic animals: Journal of the American Veterinary Medical Association, 177, 885-893.

Fowler, M., 1993, Foot care in elephants, in Fowler, M. (ed.), Zoo and wild animal medicine. Current therapy, third edition: Philadelphia/ London/Toronto, W.B. Saunders Company, 448-453.

Fowler, M., 2001, An overview of foot conditions in Asian and African elephants, in Csuti, B., Sargent, E.L., Bechert, U.S. (eds.), The elephant's foot: Ames, Iowa, U.S.A., Iowa State University Press, 3-7.

Gervais, H., 1855, Recherches sur les mammifères fossiles de l'Amérique Méridionale, in Castelnau, F., Animaux nouveaux ou rares recueillis pendant l'expédition dans les parties centrales de l'Amérique du Sud: Paris, France, P. Bertrand, 1, 1-63.

Gervais, H., Ameghino, F., 1880, Les mammifères fossiles de l'Amérique du Sud: Paris, France, F. Savy, 225 p.

Gray, J., 1821, On the natural arrangement of vertebrate animals, in Uwins, D., Palmer, S., Gray, S.F. (eds.), The London Medical Repository, monthly journal, and review: London, England, Thomas and George Underwood, 15(85), 296-310. 
Hanna, R., 2002, Multiple injury and infection in a sub-adult theropod dinosaur Allosaurus fragilis with comparisons to allosaur pathology in the Cleveland-Lloyd Dinosaur Quarry collection: Journal of Vertebrate Paleontology, 22(1), 76-90.

Haubold, H., 1996, Ichnotaxonomie und Klassifikation von Tetrapodenfährten aus dem Perm: Hallesches Jahrbuch für Geowissenschaften, B(18), 23-88.

Hay, O., 1922, Further observations on some extinct elephants: Proceedings of the Biological Society of Washington, 35, 97-102.

Henriques, D., Soares, A., Mello, M., 1998, Registros de reaçâo óssea em Panochthus Burmeister, 1866 do Pleistoceno do Estado de Paraíba: Acta Geologica Leopoldensia, 21, 149-155.

Hitchcock, E., 1844, Report on ichnolithology, or fossil footmarks, with a description of several new species, and the coprolites of birds, from the valley of Connecticut River, and of a supposed footmark from the valley of Hudson River: American Journal of Science and Arts, 47(2), 292-321.

Hitchcock, E., 1858, Ichnology of New England. A Report on the Sandstone of the Connecticut Valley, Especially its Fossil Footmarks: Boston, Massachusetts, U.S.A., W. White, 220 p.

Ishigaki, S., 1986, Dinosaur footprints of the Atlas Mountains: Nature Study (Japan), 32 (1), 6-9. Ishigaki, S., 1988, Les empreintes de dinosaures du Jurassique Inférieur du Haut Atlas central Marocain: Notes Service Geologique du Maroc, 44(334), 79-86.

Isidro, A., Malgosa, A., 2003, Paleopatología. La enfermedad no escrita: Barcelona, España, Masson, $351 \mathrm{p}$.
Jenny, J., Josen, J., 1982, Decouverte d'empreintes de pas de dinosauriens dans le Jurassique Inférieur (Pliensbachien) du Haut-Atlas central (Maroc): Compte Rendus de l'Académie des Sciences de Paris, 294(2), 223-226.

Kuntze, A., 1972, Nageltritt (Pododermatitis traumatica profunda purulenta) bei Trampeltier (Camelus bactrianus) und Elefant (Elephas maximus), in Verhandlungsbericht des 14 Internationalen Symposiums über die Erkrankungen der Zootiere: Berlin, Germany, 291-293.

Kuntze, A., 1980, Erkrankungen der Gliedmassen beim Elefanten (Elephas maximus) unter besonderer Berücksichtigung des Hufes, in Verhandlungsbericht des 22 Internationalen Symposiums über die Erkrankungen der Zoo- und Wildtiere: Arnheim, Germany, 247-253.

Linnaeus, C., 1758, Systema naturae per regna tria naturae, secundum classes, ordines, genera, species, cum characteribus, differentiis, synonymis, locis: Stockholm, Sweden, tenth edition, Laurentii Salvii, 824 p.

Lockley, M., Hunt, A., Moratalla, J., Matsukawa, M., 1994, Limping dinosaurs? Trackway evidence for abnormal gaits: Ichnos, 3(3), 193-202.

Lü, J., Kobayashi, Y., Lee, Y., Ji, Q., 2007, A new Psittacosaurus (Dinosauria: Ceratopsia) specimen from the Yixian Formation of Western Liaoning, China: The first pathological psittacosaurid: Cretaceous Research, 28(2), 272-276.

Lucas, S., 2001, Taphotaxon: Lethaia, 34(1), 30.

Lucas, S., 2005, Tetrapod ichnofacies and ichnotaxonomy: Quo vadis?: Ichnos, 12, 157-162. 
Lucas, S., Hunt, A., 2007, Ichnotaxonomy of Camel Footprints, in Lucas, S., Spielmann, J., Lockley, M. (eds.), Cenozoic Vertebrate Tracks and Traces: Albuquerque, New Mexico, U.S.A., New Mexico Museum of Natural History and Science Bulletin, 42, 155-168.

Lucas, S., Allen, B., Morgan, G., Myers, R., Love, D., Bustos, D., 2007, Mammoth footprints from the Upper Pleistocene of the Tularosa Basin, Doña Ana County, New Mexico, in Lucas, S., Spielmann, J., Lockley, M. (eds.), Cenozoic Vertebrate Tracks and Traces: Albuquerque, New Mexico, U.S.A., New Mexico Museum of Natural History and Science Bulletin, 42, 149-154.

Lull, R., 1953, Triassic life of the Connecticut Valley (revised): State Geological and Natural History Survey of Connecticut Bulletin, 81, $336 \mathrm{p}$.

Lund, P., 1840, Blik paa Brasiliens Dyreverden for Sidste Jordomvaeltning, Tredie Afhandling, Fortsaettelse af Pattedyrene: Det Kongelige Danske Videnskabernes Selskabs Naturvidenskabelige og Mathematiske Afhandlinger, 8, 217-272.

Lund, P.W., 1846, Meddelelse af det Udbytte de i 1844 undersøgte Knoglehuler have afgivet til Kundskaben om Brasiliens Dyreverden før sidste Jordomvæltning: Det Kongelige danske Videnskabernes Selskabs Skrifter, 12, 1-94.

Manera de Bianco, T., Aramayo, S., 2003, Primer registro de huellas de Equidae en el yacimiento paleoicnológico de Pehuen Co (Pleistoceno tardío) Provincia de Buenos Aires, Argentina: Ameghiniana 40(4), Suplemento Resúmenes, $61 \mathrm{R}$.

Manera de Bianco, T., Aramayo, S., 2013, Paleoichnological site of Pehuen Co (Late Pleistocene), Buenos Aires Province: mammal, bird and hominid evidence, in Melchor, R., Umazano, A. (eds.), Second
Latin American Symposium on Ichnology (SLIC 2013), Fieldtrip Guidebook: Santa Rosa, Argentina, 65-74.

Manera de Bianco, T., Bastianelli, N., Aramayo, S., 2010, Nuevos registros de icnitas de mamíferos pleistocenos en Playa del Barco, Pehuen Co, Provincia de Buenos Aires, Argentina, in Ballent, S., Artabe, A.E., Tortello, M.F. (eds.), X Congreso Argentino de Paleontología y Bioestratigrafía, VII Congreso Latinoamericano de Paleontología, Libro de Resúmenes: La Plata, Argentina, Universidad Nacional de La Plata, Museo de La Plata, 78.

Marshall, C., Brinkman, D., Lau, R., Bowman, K., 1998, Fracture and osteomyelitis in PII of the second pedal digit of Deinonychus antirrhopus (Ostrom) an Early Cretaceous 'raptor' dinosaur: Palaeontology Newsletter, $39,16$.

McCrea, R., Buckley, L., Farlow, J., Lockley, M., Currie, P., Matthews, N., Pemberton, G., 2014, A "Terror of Tyrannosaurs": The First Trackways of Tyrannosaurids and Evidence of Gregariousness and Pathology in Tyrannosauridae: PLoS ONE, 9(7), e103613.

McCrea, R., Tanke, D., Buckley, L., Lockley, M., Farlow, J., Xing, L., Matthews, N., Helm, C., Pemberton, S., Breithaupt, B., 2015, Vertebrate Ichnopathology: Pathologies Inferred from Dinosaur Tracks and Trackways from the Mesozoic: Ichnos, 22(34), 235-260.

McNeil, P., Hills, L., Tolman, M., Kooyman, B., 2007, Significance of Latest Pleistocene tracks, trackways and trample grounds from southern Alberta, Canada, in Lucas, S., Spielmann, J., Lockley, M. (eds.), Cenozoic Vertebrate Tracks and Traces: Albuquerque, New Mexico, U.S.A., Museum of Natural History and Science Bulletin, 42, 209-223. 
Mikota, S., Sargent, E., Ranglack, G., 1994, The musculoskeletal system, in Mikota, S., Sargent, E., Ranglack, G. (eds.), Medical management of the elephant: West Bloomfield, Michigan, U.S.A., Indira Publishing House, 137-150.

Miller, G., 1924, A second instance of the development of rodent-like incisors in an artiodactyl: Proceeding of the United States National Museum, 66(2545), 1-3.

Minter, N., Braddy, S., Davis, R., 2007, Between a rock and a hard place: arthropod trackways and ichnotaxonomy: Lethaia, 40(4), 365-375.

Molina, G., 1782, Saggio Sulla Storia Naturale del Chili: Bologna, Italy, Tommaso d'Aquino Stamperia, $369 \mathrm{p}$.

Molnar, R., 2001, Theropod paleopathology: A literature survey, in Tanke, D., Carpenter, K. (eds.), Mesozoic Vertebrate Life: New Research Inspired by the Paleontology of Phillip J. Currie: Bloomington, Indiana, U.S.A., Indiana University Press, 337-363.

Müller, O., 1776, Zoologiae Danicae prodromus: seu Animalium Daniae et Norvegiae indigenarum characteres, nomina, et synonyma imprimis popularium: Copenhagen, Denmark, Typiis Hallageriis, 282 p.

Oliva, C., Arregui, M., 2016, Evidences of ungual disorders in fossil tracks of Holarctic ungulates: gomphotheres (Proboscidea, Gomphotheriidae), equids (Perissodactyla, Equini) and camelids (Artiodactyla, Lamini); ichnotaxonomic implications, in XI Congreso de la Asociación Paleontológica Argentina (CAPA XI), Libro de resúmenes: General Roca, Argentina, 72-73.

Oliva, G., Arregui, M., Lirusso, V., de Valais, S., 2013, Laguna del Monte, un nuevo yacimiento paleoicnológico del Pleistoceno tardío (Piso/Edad Lujanense), Guaminí, Provincia de Buenos Aires, Argentina, in
Segundo Simposio Latinoamericano de Icnología (SLIC II), Libro de resúmenes, Santa Rosa, Argentina, 52.

Ortner, D., 2003a, Methods used in the analysis of the skeletal lesions, in Ortner, D. (ed.), Identification of Pathological Conditions in Human Skeletal Remains: San Diego, California, U.S.A., Academic Press, 45-63.

Ortner, D., 2003b, Osteoarthritis and diffuse idiopathic skeletal hyperostosis, in Ortner, D. (ed.), Identification of Pathological Conditions in Human Skeletal Remains: San Diego, California, U.S.A., Academic Press, 545-550

Ortner, D., Putschar, W., 1980, Identification of pathological conditions in human skeletal remains: Washington, U.S.A., Smithsonian Institution Press, 479 p.

Owen, R., 1848, Description of teeth and portions of jaws of two extinct anthracotheroid quadrupeds (Hyopotamus vectianus and $H$. bovinus) discovered by the Marchioness of Hastings in the Eocene deposits on the NW coast of the Isle of Wigth, with an attempt to develop Cuvier's idea of the classification of pachyderms by the number of their toes: Quaterly Journal of the Geological Society of London, 4, 103-141.

Palmer, T., 1897, Notes on the nomenclature of four genera of tropical American mammals: Proceedings of the Biological Society of Washington, 11, 173-174.

Panin, N., Avram, E., 1962, Noi urme de vertebrate in Miocenul Subcarpatilor rominesti: Studii si Cercetari de Geologie, 7, 455-484.

Peabody, F., 1948, Reptile and amphibian trackways from the Moenkopi Formation of Arizona and Utah: Bulletin Department of Geological Sciences, University of California, 27, 295-468. 
Pollitt, C., 1995, Color Atlas of the Horse's Foot: London, England, Mosby-Wolfe, Times Mirror International Publishers Limited, 208 p.

Ramsay, E., Henry, R., 2001, Anatomy of the elephant foot, in Csuti, B., Sargent, E.L., Bechert, U.S. (eds.), The elephant's foot: Prevention and Care of Foot Conditions in Captive Asian and African Elephants: Ames, Iowa, U.S.A., Iowa State University Press, 9-12.

Rega, E., Holmes, R., Tirabasso, A., 2010, Habitual locomotor behavior inferred from manual pathology in two Late Cretaceous chasmosaurine ceratopsid dinosaurs, Chasmosaurus irvinensis (CMN 41357) and Chasmosaurus belli (ROM 843), in Ryan, M., Chinnery-Allgeier, B., Eberth, D. (eds.), New Perspectives on Horned Dinosaurs, The Royal Tyrrell Museum Ceratopsian Symposium: Bloomington, Indiana, U.S.A., Indiana University Press, 340-354.

Remeika, P., 1999, Identification, stratigraphy, and age of Neogene vertebrate footprints from the Vallecito-Fish Creek Basin, AnzaBorrego Desert State Park, California: San Bernardino County Museum Association Quarterly, 46(2), 37-45.

Remeika, P., 2001, The Fish Creek Canyon Ichnofauna: A Pliocene (Blancan) vertebrate footprints assemblage from Anza-Borrego Desert State Park, California, in Santucci, V., McClelland, L. (eds.), Proceedings of the 6th Fossil Resource Conference: Lakewood, Colorado, U.S.A., Geologic Resources Division Technical Report, 55-75.

Remeika, P., Jefferson, G., Murray, L., 1995, Fossil vertebrate faunal list for the VallecitoFish Creek and Borrego-San Felipe Basins, Anza-Borrego Desert State Park and vicinity, California, in Remeika, P., Sturz, A. (eds.), Paleontology and Geology of the Western Salton Trough Detachment, Anza-Borrego
Desert State Park, California: San Diego, Association of Geologists, Field Trip Guidebook, I, 82-93.

Reynolds, R., 2006, Horse Hoof Prints in the Fossil Record, in Reynolds, R. (ed.), Making Tracks Across the Southwest, the 2006 Desert Symposium: California, U.S.A., California State University, 25-28.

Rothschild, B., 1988, Stress fracture in a ceratopsian phalanx:Journal of Paleontology, 62(2), 302-303.

Rothschild, B., 1990, Radiologic assessment of osteoarthritis in dinosaurs: Annals of Carnegie Museum, 59(4), 295-301.

Rothschild, B., 1997, Dinosaurian Paleopathology, in Farlow, J., Brett-Surman, M. (eds.), The Complete Dinosaur: Bloomington, Indiana, U.S.A., University of Indiana Press, 426-448. Rothschild, B., Martin, L., 1993, Paleopathology, Disease in the Fossil Record: Boca Raton, Florida, CRC Press, 386 p.

Rothschild, B., Martin, L., 2006, Skeletal Impact of Disease: Albuquerque, New Mexico, U.S.A., New Mexico Museum of Natural History and Science, Bulletin, 33, 1-226.

Rothschild, B., Molnar, R., 1988, Osteoarthritis in fossil marsupial populations of Australia: Annals of Carnegie Museum, 57, 155-158.

Rothschild, B., Shelton, S., 1992, Paleopathology: Austin, University of Texas, Museum of Natural History, 226 p.

Rothschild, B., Tanke, D., 1992, Paleopathology of vertebrates: Insights to lifestyle and health in the geological record: Geoscience Canada, 19(2), 73-82.

Rothschild, B., Tanke, D., 2005, Theropod paleopathology: state-of-the-art review, in Carpenter, K. (ed.), The Carnivorous Dinosaurs: Bloomington, Indiana, U.S.A., Indiana University Press, 351-365.

Rothschild, B., Tanke, D., Carpenter, K., 1997, Tyrannosaurs suffered from gout: Nature, 387, 357. 
Rothschild, B., Tanke, D., Ford, T., 2001, Theropod stress fractures and tendon avulsions as a clue to activity, in Tanke, D., Carpenter, K. (eds.), Mesozoic Vertebrate Life, New Research Inspired by the Paleontology of Phillip J. Currie: Bloomington, Indiana, U.S.A., University of Indiana Press, 331-336.

Rothschild, B., Xiaoting, Z., Martin, L., 2012, Osteoarthritis in the early avian radiation: Earliest recognition of the disease in birds: Cretaceous Research, 35, 178-180.

Santucci, V., Nyborg, T., 1999, Paleontological resource management, systematic recording, and preservation of vertebrate tracks within Death Valley National Park, California, in Desert Research Symposium, 1999: San Bernardino County Museum Press, 12-17.

Santucci, V., Nyborg, T., Buchleim, P., Nick, K., 2011, Vertebrate paleontological resources of Death Valley National Park, California, Field Trip Guide Book, in Bonde, J.W., Milner, A.R.C. (eds.), Field Trip Guide Book $71^{\circ}$ Annual Metting of the Society of Vertebrate Paleontology, 2011: Carson City, Nevada, U.S.A., Nevada State Museum, 127-155.

Sarjeant, W., Reynolds, R., 1999, Camel and horse footprints from the Miocene of California: San Bernardino County Museum Association Quarterly, 46, 3-18.

Schulp, A., 2002, The effects of tectonic deformation on dinosaur trackway morphology: Sargetia, Acta Musei Devensis, Series Scientia Naturae, Deva, 19, 27-32.

Schulp, A., Brokx, W., 1999, Maastrichtian sauropod footprints from the Fumanya Site, Berguedà, Spain: Ichnos, 6(4), 239-250.

Scrivner, P., 1984, Stratigraphy, sedimentology and vertebrate ichnology of the Cooper Canyon Formation (Neogene), Death Valley National Monument: Los Angeles, California, U.S.A.,
University of Southern California, Master of Science thesis, $355 \mathrm{p}$.

Scrivner, P., Bottjer, D., 1986, Neogene avian and mammalian tracks from Death Valley National Monument, California: Their context, classification and preservation: Palaeogeography, Palaeoclimatology, Palaeoecology, 57, 285-331.

Sullivan, R., Tanke, D., Rothschild, B., 2000, An impact fracture in an ornithomimid Ornithomimosauria:

Dinosauria) metatarsal from the Upper Cretaceous (Late Campanian) of New Mexico, in Lucas, S.G., Heckert, A.B. (eds.), Dinasours of New Mexico: Albuquerque, New Mexico, U.S.A., New Mexico Museum of Natural History and Science Bulletin, 17, 109-111.

Tanke, D., 1989, Paleopathologies in Late Cretaceous hadrosaurs (Reptilia: Ornitischia) from Alberta, Canada: Journal of Vertebrate Paleontology, 9(Supplement 3), 41A.

Tanke, D., Currie, P., 2000, Head-biting in theropods: Paleopathological evidence, in Perez-Moreno, B., Holtz, T.jr., Sanz, J., Moratalla, J. (eds.), Aspects of theropod paleobiology: Gaia, 15, 167-184.

Tanke, D., Rothschild, B., 2002, Dinosores: An annotated bibliography of dinosaur paleopathology and related topics - 1838 2001: Albuquerque, New Mexico, U.S.A., New Mexico Museum of Natural History and Science, Bulletin, 20, $97 \mathrm{p}$.

Tanke, D., Rothschild, B., 2010, Paleopathologies in Albertan ceratopsids and their behavioral significance, in Ryan, M., Chinnery-Allgeier, B., Eberth, D. (eds.), New Perspectives on Horned Dinosaurs, The Royal Tyrell Museum Ceratopsian Symposium: Bloomington, Indiana, U.S.A., Indiana University Press, 355-384. 
Tanke, D., Rothschild, B., 2015, Paleopathology in Late Cretaceous Hadrosauridae from Alberta, Canada, in Eberth, D., Evans, D. (eds.), Hadrosaurs: Bloomington, Indiana, U.S.A., Indiana University Press, 540-571.

Tassara, D., Aramayo, S., Osterrieth, M., Scian, R., 2007, Paleoicnología de mamíferos de la Formación Santa Clara (Pleistoceno tardío) en la zona costera del Partido de Mar Chiquita (Provincia de Buenos Aires) Argentina, in Actas XVI Congreso Geológico Argentino: La Plata, Argentina, 4, 219-226.

Tucker, M., Burchette, T., 1977, Triassic dinosaur footprints from South Wales: Their context and preservation: Palaeogeography, Palaeoclimatology, Palaeoecology, 22(3), 195-208.

Vialov, O., 1966, Sledy zhiznedeyatelnosti organizmov i ikh paleontogicheskoe znachenie: Kiev, Ukraine, Naukova Dumka, Akademiya Nauk Ukrainskoy SSR Institut Geologii I Geokhimii Goryuchikh Iskopayemykh, 219 p.

von Houwald, F., 2001, Foot problems in Indian rhinoceroses (Rhinoceros unicornis) in zoological garden: macroscopic and microscopic anatomy, pathology and evaluation of the causes: Zürich, Switzerland, Zürich University, $\mathrm{PhD}$ thesis, $104 \mathrm{p}$.
Waldron, T., 2009, Paleopathology: New York, U.S.A., Cambridge University Press, 279 p.

Webb, S., 1965, The osteology of Camelops: Bulletin of the Los Angeles County Museum of Natural History, 1, 1-54.

West, G., 2001, Ocurrence and treatment of nail/ foot abscesses, nail cracks and sole abscesses in captive elephants, in Csuti, B., Sargent, E.L., Bechert, U.S. (eds.), The elephant's foot: Ames, Iowa, U.S.A., Iowa State University Press, 93-97.

Xing, L., Dong, H., Peng, G., Shu, C., Hu, X., Jiang, H., 2009, A scapular fracture in Yang-chuanosaurus hepingensis (Dinosauria: Theropoda): Geological Bulletin of China, 28(10), 1390-1395.

Xing, L., Rothschild, B., Ran, H., Miyashita, T., Persons, W., Sekiya, T., S., Zhang, J., Wang, T., Dong, Z., 2015, Vertebral fusion in two Early Jurassic sauropodomorph dinosaurs from the Lufeng Formation of Yunnan, China: Acta Paleontologica Polonica, 60(3), 643-649.

Zittel, K., 1893, Palaeozoologie, IV, Mammalia in Zittel, K., Handbuch der Palaeontologie: Munich and Leipzig, Germany, Oldenbourg, 799 p.

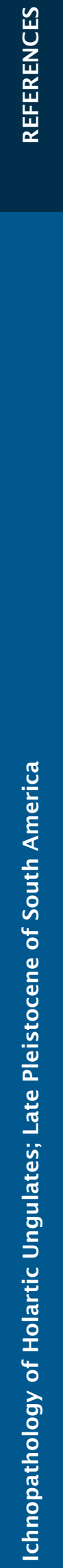

